\title{
XVI.
}

\section{Die Topographie des Krebses. \\ (Dritte Mitteilnng des Bayerischen Komitees für Krebsforschung.)}

Untersuchungen anf Grund der Bayerischen Krebssterblichkeit.

Von

Karl Kolb, Schriftfihrer.

(Mierzu Tafeln 1II--VI.)

I.

Bis vor kurzem war eine der umstrittensten Fragen der Krebsätiologie die Frage der Erblichkeit, obwohl schon lange einzelne Beobachtungen, wie die von Weinberg und Gastpar (1) einen erblichen Einfluss hatten aumehmen lassen. Diese Annahme wurde aber immer wieder anf Grund subjektiver Ansichten ohne Prüfung verneint.

Aehnlich stand es, ja steht es heute noch mit der Frage: Gibt es tatsächliche Verschiedenheiten der Häufigkeit des Krebses in den einzelnen Ländern oder an einzelnen Orten, obwohl diese Frage nach so vielen bekannt gewordenen Tatsachen, ron den Untersuchungen Havilands im Jahre 1868 an, schon längst beantwortet sein dürfte. Und doch stossen wir auch hier auf dieselbe Erscheinung, dass trotzdem leichthin Ausspriiche getan werden, wie der Bashfords (2), dass die Häufigkeit des Krebses mit der ärztlichen Kultur zusammenhängt und darum in der Schweiz, wo die Leichenschau eingeführt ist, so gross, und in Preussen, wo sie nicht obligatorisch von den Aerzten vorgenommen wird, viel geringer ist.

Die einzige Entschuldigung für derartige Meinungsäusserungen (etwas anderes sind sie nicht) geben viele mangelhafte statistische Arbeiten über die Topographie des Krebses. Aber es fehlten doch auch schon längst nicht Arbeiten, welche den früheren Fehler der Nichtberïcksichtigung des Alters vermieden haben. So hatte meine erste Untersuchung über das Vorkommen des Krebses in Süddeutschland (3) die Sterblichkeit überall lür eine Berölkermng jenseits des 40. Lebensjahres berechnet.

Seit 190ら wurde die selbständige Bearbeitung des statistischen Urmaterials in Bayern von dem dortigen Krebskomitee ganz besonders zu lem Zwecke aufgenommen, eine möglichst genaue Krebstopographie des

Zeitschrift für Krebsforschung. 14. Bd. 2. Heft. 
Landes herzustellen und zwar unter Yermeidung der bisherigen Fehler. Es sollte das Alter nicht nur insoweit berücksichtigt werden, dass nur die Berölkerung der höheren Lebensjahre, z. B. über dem to. oder 30, wie in Deutsehland zuerst ron Laspeyres (t), als Masstab der Krebssterblichkeit dienen sollte. Da der Altersaulbau der Bevölkermo über 30 Jahren in den einzelnen Ländern, Kreisen und Stüdten sehr verschieden ist, sollte rielmehr zunächst die Sterblichkeit für die einzelnen Altersklassen dieser Bevölkerung gesondert berechnet werden. Das ist schon in den flüheren Mitteilungen des Bayerischen Krebskomitees geschehen. Weil aher diese Sterblichkeitszahien nicht in eine Gesamtzahl für das betreffende Gebiet zusammenzufassen und für eine kartographische Darstellung geeignet sind. musste weiter die Berechunng nach einer Standardbevölkerung; wie sie $O g l e$ und Körosy vorgeschlagen haben, hinzugefïgt werden. Es wurde also zuerst die Sterblichkeit der drei Altersklassen von 30-50, 50-70 und von 70 und mehr Jahren für die einzehnen Verwaltungsibezirke berechnet und dann deren Gesamtsterblichkeit berechnet unter der Annahme, dass ihre Berölkerung iilser 30 .Jahren genan donselben Altersaubau hätte, wie die Gesamberölkerung Bayens.

Ein zweiter Fehler, welcher last immer das statistische Ergebnis namentlich fiur die Städte fälscht, ist die Nichtausscheidung der von auswärts zngezogenen Kranken. Diese geschah bis jetzt nur in der Schweiz, in Schottland, in Norwegen seit 1892. in England für die stattiche, nicht die lokale Statistik. Sie geschah auch für das ganze Material der vorliegenden Arbeit. Trotz der betreffenden Ministerialentschliessung wird allerdings die Angabe des Wohnortes neben dem des Sterbeortes rou den Leichenschanem noch sehr unvollstandig ausgefüht, so gerade viedfach ron seiten der grossen Trankenhänser; doch haben unermidlich fortgesetzte Nachfragen alljählich die Auswätigen namentlich der Kraulienhäuser rollstundig adsscheiden und inen truheren Wohnorte zuscheiben lassen. Freilich muss zugegeben werden. dass noch manche zur Pflege und Behandlung Zugezogene. welche in Privathïusern, z. B. bei Terwandten gestorthen sind, so lange nicht als solche erfasst werden können, bis clie Leichenschnuer auch in Privathäuserm strenge die Verordnung der Angabe les Wohnortes befolgen. Tmmerhin ist der Hauptfehler durch Richtigstellung der in Krankenhätsern Verstorbenen numehr beseitigt. Als Massstab lür die Grösse dieses Fehlers sci mur angeführt, dass er in der ganzen Berichtszeit für Hünchen 10 pCt., für Nüirzburg 1911 über $1 / 3$, für Erlangen 190 sar über die Hälfte aller Krebstoten ausmachte.

Alles Material, welches zu mnerer Untersuchnng benutzt wurde, d. h. die sämtlichen Leichenschauscheine ron an Frebs in Bayen Gestorbenen wälnend der 7 Jahre 1905-1911 lagen dem Verpasser vor und wurden von ihm allein beabeitet. Es handelt sich um 5022:3 Tudeslälle, 21!3.5 bei 
Männern, 28238 bei Frauen. Die Zahlen sind so gross, dass sie für grössere Verwaltungsbezirke: Regierungsbezirke; Bezirksämter und grössere Städte zu vollständig sicheren Ergebnissen genügen, ja sie hätten sogar noch zur Berechnung der Sterblichkeit der kleineren Amtsgerichtsbezirke bemutzt werden können.

Eine Trennung zwischen Carcinom und Sarkom wurde dabei nicht mehr vorgenommen, weil sie keine ätiologische Bedeutung hat.

Wie soll nun eine Topographie des Krebses hergestellt werden? Soll sie, wie es bisher schon aus Mangel an genauen Unterlagen gewöhnlich geschah, nur die Krebssterblichkeit im Ganzen geben oder nur je die Sterblichlseit der einzelnen Lokalisationen, wie es von Vielen, am schärfsten ron Ewing (6) verlangt wird? Er sagt: "Deshalb scheint mir, dass statistische Studien auf die einzelnen Typen (d. h. Lokalisationen) des Krebses beschrünkt werden sollten und dass wir die Neigung, alle Krebse als eine Krankheit zu klassifizieren, rermeiden sollten. Ich sehe keinen Nutzen im Zusammenfassen von Hodenkrebs mit Krebs der Lippe oder Zunge."

Ich bin durchaus nicht dieser Ansicht. Ich halte die Untersuchung der einzelnen Lokalisationen für durchaus notwendig, aber die der Gesamtsterblichkeit an Krebs fïr noch notwendiger und habe dies schon früher ( $(\tau)$ begründet. Erkrankung und Tod an Krebs ist, wie ich dort ausgeführt habe, anerkanntermassen nicht Folge einer Ursache, wie z. B. bei einem Trauma, bei welchem iibrigens die Körperbeschaffenheit des Verletzten auch von Einfluss ist, sondern Folge des Zusammenwirkens einer Reihe ron "Koeffizienten" nach Hering (\&) oder „Bedingungen" nach Hansemann (9).

Wir können nicht durch örtlichen Reiz allein die Entstehung des Krebses erklären; es gehört dazu die Disposition, dem sonst müsste der Reiz in jüngeren Jahren ebenso leicht Krebs hervorrufen, wie im Alter, und wenn wir alle annehmen, dass der Krehs von einer umschriebenen Stelle des Körpers, einer Zelle oder Zellgruppe, ausgeht, so brauchen wir darum noch nicht $\mathrm{zu}$ schliessen, dass das, was wir Disposition nennen, sich auch immer nur aul eine einzige Stelle beschränke; wir werden rielmehr auch eine allgemeine Disposition annehmen müssen. Dafür sprechen die Beobachtungen von primär multiplen bösartigen Neubildungen, von denen z. B. Hans Hauser (10) berichtet, sogar von besonderer Neigung zu gutartigen Tumoren olme Neigung zu bösartigen Tumoren nach $K \ddot{K} \ddot{l} \mathrm{z}$ (11), manche späte Rezidive, welche eher als neue Entstehungen in einem disponierten Körper aufzufassen sind, dafür der Einfluss der Erblichkeit des Krebses, welcher sich bei den Nachkommen zwar oft, aber nicht immer in demselben Organ wiederholt, dafür die [ntersuchungen ron E. Freund. Es gibt allgemeine "Bedingungen", 
wie das Alter, welches gewiss nicht nur in einzelnen Zellen eine Disposition schafft und bei welcher nur der Angriffspunkt des zuerst wirksamen Reizes den primüren Sitz der Erkrankung bestimmt. Um diese Sumne ron Agentien zu entwirren, brauchen wir zur ätiologischen Beurteilung der Sterblichkeit an den einzelnen Lokalisationen zuerst die Feststellung der Gesamtsterblichkeit an Krebs.

Tabelle I gibt eine Lebersicht der Krebstodesfälle und des Altersunfbaues in den einzelnen Regierungsbezirken Bayerns nach der Tolkszällung. von 1910. Für die gütige Mitteilung des Altersaufbaues bin ich dem Vorstande des Kgl. Statistischen Landesamtes, Hern Ministerialrat Zahn, zu Danke verpflichtet. Dabei ist aus später zu erörternden Gründen Niederbayern in einen Teil nördlich der Donau und eimen Teil siidlich der Donau, letzterer das ganze Bezirksamt Kelheim einschliessend, geteilt.

Tabelle I.

a) Männer.

\begin{tabular}{|c|c|c|c|c|c|c|c|c|c|}
\hline \multirow{2}{*}{$\begin{array}{c}\text { Regierungs- } \\
\text { bezirk }\end{array}$} & \multicolumn{5}{|c|}{ Krebstodesfälle } & \multicolumn{4}{|c|}{ Bevö]kerungszahl } \\
\hline & 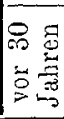 & 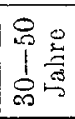 & $\underset{0}{0} \stackrel{0}{0}$ & 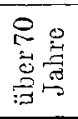 & Alle & $\begin{array}{l}80 \\
10 \\
10 \\
0\end{array}$ & $\stackrel{0}{1}$ & 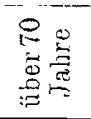 & $\begin{array}{c}\text { Gesamt- } \\
\text { bevolke- } \\
\text { rong }\end{array}$ \\
\hline & & $\$ 91$ & 3273 & 1325 & & & 93028 & 18079 & \\
\hline $\begin{array}{l}\text { Niederb } \\
\text { (siidl. d }\end{array}$ & 3 & 204 & 902 & 421 & 60 & 29 & 33997 & 8400 & 078 \\
\hline $\begin{array}{l}\text { Niede } \\
\text { (nor }\end{array}$ & 17 & 68 & 272 & 126 & 473 & 24677 & 15058 & 4065 & 111571 \\
\hline Pfal & 49 & 275 & 1450 & 587 & 2361 & 109542 & 51936 & 10361 & 91 \\
\hline & 18 & 235 & 1173 & 399 & 25 & 65694 & 39 & 8987 & 397 \\
\hline & 2 & 22 & 1131 & 380 & 1759 & & & & \\
\hline & 7 & $4=$ & 1648 & 63 & 2796 & 119 & 54 & 10 & 05 \\
\hline & 6. & 270 & 1308 & 603 & 2942 & & 46 & 10538 & \\
\hline & & 420 & 2060 & 882 & 3370 & 94816 & 51928 & 11173 & 389972 \\
\hline 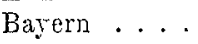 & 134 & 3085 & 13217 & 5309 & 21985 & 829446 & 27488 & 91261 & 3379580 \\
\hline
\end{tabular}

b) Frauen.

\begin{tabular}{|c|c|c|c|c|c|c|c|c|c|}
\hline Oberbayern & 156 & 1677 & 4208 & 1657 & 7698 & 204659 & 106632 & 24464 & 780288 \\
\hline $\begin{array}{l}\text { Niederbayern } \\
\text { (siidl. d. Donau) }\end{array}$ & 33 & 4111 & 1199 & 516 & 2159 & 57228 & 36678 & 9307 & 253937 \\
\hline $\begin{array}{l}\text { Niederbajern } \\
\text { (nördl. d.Donan) }\end{array}$ & 15 & 169 & 314 & 152 & 650 & 25254 & 16160 & 4578 & 115745 \\
\hline Pfalz. & 55 & 479 & 1762 & 722 & 3018 & 110404 & 57718 & 12295 & 473394 \\
\hline Oberpfalz & 31 & 387 & 1247 & 443 & 2108 & 69306 & 43814 & 10498 & 87 \\
\hline Oberfrauken. & 33 & 407 & 1275 & 435 & 2150 & $808+2$ & 48072 & 11219 & 33 \\
\hline ken & 66 & 789 & 2062 & 815 & 3732 & 122478 & 63861 & 14025 & 476663 \\
\hline ken & 63 & 459 & 1583 & 618 & 2723 & 88900 & 52559 & 11916 & 362951 \\
\hline Schwaben & 49 & 728 & 2248 & 975 & 4000 & 95321 & 58047 & 13910 & 399881 \\
\hline Bayern & 501 & 5506 & 15898 & 6333 & 28238 & 854392 & 483541 & 112012 & 3507711 \\
\hline
\end{tabular}


Ehe wir weitergehen, soll vor allem die Frage erörtert werden: Sind die zugrunde liegenden Angaben der Leichenschauscheine hinreichend vertrauenswürdig, um zu topographischen Vergleichen benutzt werden zu können? Ausser dem früher (12) über die Bayerische Sterblichkeitsstatistik Gesagten ist darauf zu erwidern:

Die Vorschriften über die Vornahme der Leichenschau sind in ganz Bayern dieselben, und wenn anch die Genauigkeit der Erhebungen von manchen örtlichen Umständen, die nirgends ganz gleich sein können, beeinflusst wird, namentlich von der Häufigkeit der vorausgegangenen ärztlichen Behandlung, der Gewissenhaftigkeit der Leichenschauer, der Sorgfalt der Bezirksärzte, so kann doch ausgesprochen werden, dass die Unterlagen unserer Statistik im wesentlichen für alle Teile des Landes gleichwertig sind. Nur die zwei Regierungsbezirke Oberpfalz und Niederbayern dürften in der Vollständigkeit nicht ganz den andern gleichkommen, insofern bei diesen wohl die Zahl der ïrztlich behandelten unter den Krebskranken nahezu gleich gross ist, wie in den übrigen Regierungsbezirken (1910: 93,2 und $93,7 \mathrm{pCt}$. gegen 92,6 bis $98,5 \mathrm{pCt}$.), aber unter allen überhaupt Gestorbenen in der Oberpfalz nur 53, in Niederbayern nur 54 ärztlich bchandelt wurden gegeniiber 61 bis $82 \mathrm{pCt}$. in den andern Regierungsbezirken. Wenn auch die Unterschiede hauptsächlich auf die Todesfälle der Kinder treffen, so möchte ich meine Vermutung einer geringeren Vollständigkeit der Angaben namentlich für die Oberpfalz auch mit Rücksicht auf sonstige Verhältnisse für wahrscheinlich halten. Ich kam dafür noch den Umstand anführen, dass die Sterblichkeit auf dem Lande, wie Tabelle II zeigt, in der Oberpfalz im Verhältnis zur städtischen am geringsten von allen Regierungsbezirken ist, was zum Teil als Folge einer noch etwas mangelhafteren Erhebung, als sie sonst schon auf dem Lande ist, zu deuten sein dürfte; weiter die Annahme damit stützen, dass entgegen allen anderen Erfahrungen die Krebssterblichkeit in der höchsten Altersklasse iiber 70 Jahre in einzelnen Bezirksämtern der Oberpfalz (Eschenbach, Roding, Oberviechtach) nur wenig grösser, teilweise sogar geringer ist, als in der Altersklasse von 50-70 Jahren.

Eine zweite Fehlerquelle, auf welche in der Regel bei Aufstellung der Krebstopographie nicht geachtet wird, ist das Verhältnis der städtischen zur ländlichen Bevölkerung. Ihre Beurteilung macht es notwendig, kurz auf die Krebshäufigkeit in Stadt und Land einzugehen. In den Städten ist die Krebssterblichkeit meist grösser als auf dem umliegenden Lande, - indes nicht immer, denn ich habe schon früher (13) nachgewiesen, dass eine Reihe von bayerischen Städten eine geringere Krebssterblichkeit hat, als der umliegende Landbezirk, und dasselbe zeigen auch die unten mitgeteilten Zahlen von Würzburg und Augsburg. Ja im ganzen Regierungsbezirk Schwaben verhält sich die Krebssterblichkeit von Land und Stadt 
bei den Männern wie 98, bei den Frauen wie 93:100. Man wird in der Annahme nicht fehlgehen, dass die Sterblichkeit in diesem ganzen Regierungsbezirk auf dem Lande eine höhere ist. Sie liegt nahe, wenn wir berücksichtigen, dass die städtische Krebssterblichkeit scheinbar vergrössert wird durch die häufigere ärztliche Behandlung, dann nicht nur durch die Zugänge in die Krankenanstalten (diese Fehlerquelle ist ja für uns ausgeschieden), sondern auch durch die in Privathäuser zu Angehörigen zuziehenden Kranken. Nach den Tabellen überwiegt infolgedessen anch die städtische Krebssterblichkeit die ländliche im ganzen Königreiche und in allen Regierungsbezirken.

Tabelle II.

Krebssterblichkeit auf dem Lando gegenüber der Stadt $(=100)$.

\begin{tabular}{|c|c|c|c|}
\hline & & Männer & Frauen \\
\hline $\begin{array}{l}\text { Oberbayern . } \\
\text { Niederbayern. } \\
\text { Oberpfalz. } \\
\text { Oberfranken . } \\
\text { Mittelfranken. } \\
\text { Unterfranken. } \\
\text { Schwaben. . }\end{array}$ & 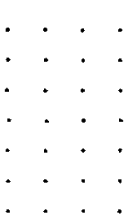 & $\begin{array}{l}85 \\
87 \\
79 \\
82 \\
83 \\
87 \\
98\end{array}$ & $\begin{array}{l}84 \\
87 \\
67 \\
72 \\
78 \\
93 \\
93\end{array}$ \\
\hline Königreich ohno & Pfalz $^{1}$ ). & 82 pCt. & 79 pCt. \\
\hline
\end{tabular}

Bei den verhältnismässig noch geringeren Zahlen der weiblichen Krrebssterblichkeit auf dem Lande liegt es nahe, an eine noch mangelhaftere Erhebung zu denken, worauf wir später zurückkommen werden.

Bei unseren späteren Untersuchungen erscheint die Yergleichung zwischen Süd- und Nordbayern hervorragend wichtig. Man könnte nun vermuten, dass der Unterschied zwischen der städtischen und ländlichen Krebssterblichkeit auf diesen Vergleich einen um so störenderen Einfluss haben wird, als die Stadtbevölkerung in Südbayern absolut ïber 3 mal so gross ist als in Nordbayern (Ober- und Unterfranken). Es stellte sich aber heraus, dass die Uebersterblichkeit im Süden gegenüber dem Norden auf dem Lande sogar noch grösser ist als in der Stadt. Setzt man für Nordbayern die Zahl 100, so hat Südbayern eine Krebssterblichkeit für die Männer in der Stadt von 124,3, auf dem Lande aber 129,2, und für die Frauen 132,6 in der Stadt, 138,3 auf dem Lande. Danach konnte der Fehler durch die grössere städtische Bevölkerung in Südbayern nicht

1) Diø Ausscheidung nach Stadt und Land fehlt für die Pfalz fast durchgehends in der bayerischen Medizinalstatistik. 
bedeutend sein, und wirklich ergab eine Berechnung, bei welcher für Nordbayern eine gleich grosse Stadtbevölkerung, wie sie Südbayern besitzt, nämlich 33,4 pCt., und eine gleich grosse ländliche Bevölkerung, 66,6 pCt., eingesetzt wurde, für die allgemeine Krebssterblichkeit in Nordbayern für die Männer die Zahl 2074 statt 1953, und das entsprechende Verhältnis zu Südbayern wie $100: 126,4$ statt $100: 130$. Für die Frauen bei 34,2 pCt. städtischer und 65,8 pCt. ländlicher Bevölkerung ist die entsprechende Verhältniszahl 136,3 gegen 136,3 .

Der Unterschied zwischen der ursprünglichen und der korrigierten Zahl ist also bei den Männern sehr gering (für den Regierungsbezirk Schwaben fast Null), bei den Frauen gar nicht vorhanden, so dass die Korrektur für die einzelnen Lokalisationen gar nicht berechnet und überhaupt nicht berücksichtigt wurde.

Kehren wir nach dieser Abschweifung wieder zum Gang der Untersuchung. zurück. Es wurde die Krebssterblichkeit der 163 Bezirksämter und der sogenannten unmittelbaren Städte für die drei früher angegebenen Altersklassen über 30 Jahre, getrennt nach beiden Geschlechtern, berechnet und die erhaltenen Zahlen wurden dann für die bayerische Durchschnittsbevölkerung als Standardbevölkerung umgerechnet (siehe S. 200).

In der folgenden Zusammenstellung sind die gemeinsamen Zahlen von Stadt und gleichnamigem Bezirksamt angegeben, mit Ausnahme der besonders angeführten Grossstädte Nünchen, Nürnberg, Angsburg und Würzburg.

Tabelle III.

Krebssterblichkeit der Bezirksämter und Städte. (Berechnung nach Ogle.)

\begin{tabular}{|c|c|c|c|c|c|}
\hline \multicolumn{2}{|c|}{ 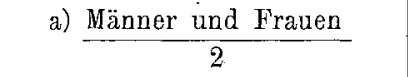 } & \multicolumn{2}{|c|}{ b) Männer } & \multicolumn{2}{|c|}{ c) Frauen } \\
\hline 1. Grafenau & 840 & 1. Grafenau. & 727 & 1. Oberviechtach & 924 \\
\hline 2. Oberviechtach & 948 & 2. Viechtach & 857 & 2. Grafenau. & 952 \\
\hline 3. Viechtach . . & 1225 & 3. Oberviechtach & 971 & 3. Wolfstein & 1421 \\
\hline 4. Königshofen & 1274 & 4. Königshofen & 1028 & 4. Regen & 1461 \\
\hline 5. Wolfstein (Nbr.) & 1316 & 5. Cham . & 1046 & 5. Königshofen & 1520 \\
\hline 6. Cham & 1364 & 6. Kötzting & 1049 & 6. Naila... & 1524 \\
\hline 7. Regen & 1444 & 7. Wolfstein & 1180 & 7. Stad tsteinach & 1532 \\
\hline 8. Stadtsteinach & 1492 & 8. Vilshofen & 1279 & 8. Eschenbach & 1563 \\
\hline 9. Bergzabern & 1541 & 9. Lichtenfels & 1323 & 9. Viechtach & 1593 \\
\hline 10. Mellrichstadt & 1548 & 10. Passau. . & 1357 & 10. Homburg & 1652 \\
\hline 11. Kötzting & 1574 & 11. Hof heim & 1368 & 11. Brückenau & 1682 \\
\hline 12. Lichtenfels & 1587 & 12. Bergzabern & 1398 & 12. Cham & 1682 \\
\hline 13. Hof heim . & 1600 & 13. Roding. & 1422 & 13. Bergzabern & 1684 \\
\hline 14. Roding. & 1606 & 14. Regen & 1427 & 14. Bogen & 1696 \\
\hline 15. Bogen & 1625 & 15. Kronach & 1444 & 15. Kemnath & 1701 \\
\hline 16. Homburg. & 1657 & 16. Stadtsteinach & 1451 & 16. Staffelstein & 1708 \\
\hline 17. Kronach & 1708 & 17. Rothenburg & 1056 & 17. Roding & 1789 \\
\hline 18. St & 1758 & 18. Bogen. & 1560 & 18. Forchheim & 1796 \\
\hline
\end{tabular}


a) Männer und Frauen 2 b) Männer c) Frauen
19. Kulmbach

20. Teusehnitz

21. Vilshofen

22. Kemnath

23. Naila.

24. Rothenburs

25. Bamberg 2

26. Brückenau

27. Stadtamhof

99.

. Richstätt.....

30. Rehau

31. Pegnitz

32. Straubing ......

33. Deggendorf

34. Zweibrücken.....

35. Karlstadt . . . . .

36. Gemünden .....

37. Berneck ......

38. Ebern .......

39. Gunzenhausen . . . .

40. Eschenbach . . . .

41. Tirsehenreuth ....

42. Sulzbach . . . . . .

43. Forchheim .

44. Neustadt (Unterfr.).

45. Germersheim ....

46. Ebermannstadt . .

47. Kitzingen

48. Scheinfeld

49. Neustadt (Oberpfalz)

50. Kissingen

ว1. Hof .

52. Hassfurt

53. Obernburg

54. Münchberg

55. Speyer.

56. Sonthofen

57. Ochsenfurt

58. Bayreuth

59. Nabburg .

60. Gerolzhofen

61. Vohenstraus

62. Mainburg

63. Landau (Pfalz) ...

64. Marktheidenfeld...

65. St. Ingbert .....

66. Höchstadt .....

67. Burglengenfeld ...

68. Feuchtwangen . . .

69. Pirmasens . . . . .

70. Eüssen . . . . . . .

71. Bruck
1780

1805

1827

1837

1853

1864

1872

1882

1883

1892

1902

1909

1910

1918

1929

1956

1965

1981

2017

2025

2035

2037

2038

2039 44. Obernburg . . . . .

2058 45. Bayreuth

2063 46. Kusel

2080 47. Neustadt (oberpaiz

9088 48. Sonthoten

2089 49. Sehweinfurt . . . . .

2091 50. Tandshut . . . . .

2093 51. Scheinteld ......

2102 52. Lauf .

2108 53. Mühldorf.

2112 54. Pegnitz

211255 . Landau (Niederb.).

2114 56. Rebau .......

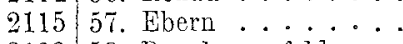

2123 58. Burglengenfold . . .

2151 59. Schwabach . . . . .

2159 60. Bez-Amt Nürnberg.

2163 61. Ludwigshafen ...

2172 62. Dingolfing .....

2177 63. Kemnath......

2194 64. Gerolzhofen .....

2199

2208

2216

2223

2231

2243

2246
1580

19. Kulmbach .... 180

1606 20. Teuschnitz..... 182

1648 21. Hof heim ...... 183]

1652 22. Lichtenfels .... 1850

1661 23. Sulzbach . . . . . 188

1687 24. Pegnitz . . . . . 188

1690 25. Kronach ....... 197

1694 26. Rehau ....... 197

1710 27. Tirschenreuth ... 198:

1733 28. Marktheidenfeld . . 202k

1744 29. Nabburg . . . . 202

1756 30. Bamberg 2 . . 203

1757 31. Mellrichstadt . . . 204

1787 32. Gemünden ...... 208:

1802 33. Kissingen ..... 208:

1807 34. Ebermannstadt . . 209:

1822 35. Kötzting ...... 209

1826 36. Feuchtwangen ... 210

1831 37. Stadtamhof .... 211

1840 38. Eichstät. . . . . 211

1843 39. Berneck ...... 211

1846 40. St. Ingbert . . . 215

1857 41. Deggendorf . . . 216

1858 42. Zweibrücken.... 216

1858 43. Rothenburg .... 217

1864 44. Pirmasens ..... 217

1879 45. Hof . . . . . 217

1884 46. Vohenstraus .... 220

1892 47. Karistadt ...... 222

1902 48. Gunzenhausen. . . 222

1911 49. Straubing . . . . . 229

1915 50. Germersheim .... 225

1918 51. Scheinfeld ...... 225

1927 52. Neustadt (Oberpfalz) 228

1928 53. Tielheim ...... 230

1932 54. Garmisch ...... 232

1936 55. Sonthofen ...... 232

1942 56. Neustadt (Unterfr.) . 233

1942 57. Gerolzhofen.... 234

1946 58. Obernburg . . . . . 235

1947 59. Mainburg . . . . . 235

1964 60. Hammelburg . . . 235

1967 61. Bruck ....... 236

1972 62. Bayreuth ..... 236

1972 63. Vilshofen ..... 237

1976 64. Münchberg. . . . . 238

1992 65. Waldmünchen .... 239

2005 66. Speyer....... 239

2009 67. Hassfurt . . . . . 240

2019 68. Kitzingen . . . . 240

2026 69. Ochsenfurt. . . . 240

2027 70. Dinkelsbühl . . . 241

2033 71. Neumarkt ..... 242 

a) Männer und Frauen
2
b) Männer
c) Frauen

72. Landshut .

73. Griesbach

2251

2254

74. Wunsiodel

75. Ludwigshafen

76. Sehweinfurt

77. Schwabach ....

78. Weilheim

79. Mühldorf . . . . . .

80. Hersbruck ....

81. Waldmünchen ...

82. Lauf

83. Hilpoitstein ....

84. Stadt Würzburg . .

85. Kelheim ......

86. Uffenheim ....

87. Ansbach

88. Bez.-Amt Nürnberg

89. Lindau .......

90. Stadt und Bez-Amt Bamberg $1 \ldots$.

91. Bez-Amt München

92. Ingolstadt .....

93. Kusel . . . . . . .

94. Kirchheimbolanden

95. Parsberg ......

96. Tölz

97. Schongau

98. Dürkheim

99. Beilngries .....

100. Dingolfing ....

101. Rockenhausen ...

102. Wolfratshausen ..

103. Wegscheid .....

104. Lohr ........

105. Garmisch

106. Neunburg

107. Dillingen

108. Fürth .

109. Ilammelburg $\cdots$

110. Regensburg ....

111. Neustadt (Mittelfr.)

112. Miesbach ......

113. Neumarkt .....

114. Wasserburg ....

115. Kaiserslautern ....

116. Bez.-AmtWürzburg

117. Neustadt (Pfalz).

118. Dinkelsbühl . . .

119. Weissenburg . .

120. Alzenau ......

121. Amberg. . . . . .

122. Landau (Niederb.).

123. Frankenthal ....
2258

2266

2273

2282

2282

2301

2303

2310

2349

2351

2353

2367

2372

2380

2381

2381

2390

2393

2402

2409

2425

2431

2441

2451

2453

2453

2461

2463

2464

2470

2504 105. Neustadt (Mittelir.)

2514 106. Beilngries

2533

2549

2560

2565

2579

2593

2621

2622

2623

2635

2644

2650

2685

2694

2698

2707

2716
72. Weilheim ..... 2057

73. Ingolstadt . . . 2062

74. Hersbruek . . . 2064

75. Brückenau .... 2082

76. Kissingen . . . . 2096

77. Laufen . . . . 2109

78. Vohenstraus . . . 2120

79. Lohr . . . . . 2124

80. Bruck . . . . . . 2131

81. Uffenheim .... 2148

82. Wolfratshausen .. 2156

83. Kirchheimbolanden 2169

84. Naila . . . . . . 2181

85. Tirschenreuth ... 2185

86. Hiltpoltstein ... 2185

87. Sulzbach ..... 2191

88. Rockenhausen ... 2200

89. Wasserburg .... 2221

90. Stadt Würzburg . 2228

91. Waldmünchen .. : 2228

92. Rottenburg. . . . 2230

93. Bamberg 1.... 2230

94. St. Ingbert . . . . 2243

95. Weissenburg . . . 2261

96. Ansbach ..... 2269

97. Nabburg . . . . 2272

98. Dürkheim .... 2273

99. Forchheim ... 2279

100. Pirmasens .... 2288

101. Traunstein .... 2298

102. Dillingen ..... 2306

2309

2328

2328

2331

2336

2344

2345

2345

2353

2372

2408

2424

2424

2427

2432

2437

2487

2546
120. Füssen ...... 2500 120. Füssen ..... 2986

121. Neunburg .... 2510 121. Krumbach .... 2992

122. Pfaffenhofen ... 2538 122. Pfaffenhofen ... 3016

72. Passau

2427

73. Parsberg ......

74. Bez.-Amt München

75. Stadt Würzburg. .

76. Burglengenfeld...

77. Wunsiedel .....

78. Ansbach .....

79. Weilheim ......

80. Hilpoltstein . . .

81. Neunburg .....

82. Hersbruck

83. Bamberg $1 \ldots .2549$

84. Höchstadt . . . 2558

85. Ludwigshafen ... 2564

86. Beilngries .... 2575

87. Landshut . . . . 2587

88. Uffenheim ... 2596

89. Schwabach .... 2617

90. Landau (Pfalz) . . 2621

91. Dürkheim ... 2633

92. Schweinfurt . . . 2635

93. Bez.-Amt Würzburg 2635

94. Griesbach .... 2650

95. Fürth . . . . . 2661

96. Riedenburg. . . . 2665

97. Mühldorı ..... 2674

98. Kirchbeimbolanden 2680

99. Rockenbausen ... 2725

100. Ingolstadt . . . 2742

101. Dillingen ..... 2760

102. Lauf. . . . . . 2770

103. Wolfratshausen . . 2772

104. Bez.-Amt Nürnberg 2797

105. Regensburg ... 2802

106. Kaiserslautern . . 2818

107. Lohr ....... 2828

108. Neustadt (Mittelfr.) 2830

109. Alzenau ..... 2837

110. Miesbach . . . . 2842

111. Tölz . . . . . . 2854

112. Frankenthal . . 2863

113. Erlangen ..... 2864

114. Schongau . . . . . 2883

115. Neu-Ulm . . . . 2899

116. Kusel . . . . . 2932

117. Wegsebeid. . . . . 2934

118. Neustadt (Pfalz). . 2935

119. Dingolfing .... 2950

123. Eggenfelden ....

$\begin{array}{lll}\text { 122. Pfaffenhofell } & \ldots & 3016 \\ \text { 123. Erding . . . . } & 3021\end{array}$ 

a) Männer und Frauen 2
b) Männer
c) Erauen

\begin{tabular}{|c|c|c|c|c|c|}
\hline 19 & & 4. 1 I:T & 47 & rburg & \\
\hline ein & & 5. Alzenau & 2551 & 25. Aiehach & \\
\hline Erl & 2 & kenthal & 2569 & enburg & \\
\hline arg & 2755 & 7. $\mathrm{Ma}$ & & 27. VI & \\
\hline fen & & 8. K & & 28. A & \\
\hline 1 & & 9. $\mathrm{Me}$ & & ïnchen & \\
\hline $\operatorname{lrg}$ & 32 & $0 . \mathrm{E}$ & & 0. & \\
\hline $\mathrm{N}$ & 7 & 1. & & burg & \\
\hline . & 1 & Würzburg & & 32. 1 & \\
\hline & & nberg & & Nürnberg & \\
\hline rnberg. . & & & & 34.1 & \\
\hline 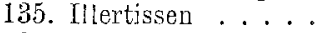 & & . 0 & & 135. 1 & \\
\hline & & & & 36. I & \\
\hline hen & & 7. 11 & & 37.7 & \\
\hline burg . . & & - St & & gsbourg & \\
\hline $\arg$ & & . R & & 39. & \\
\hline & & burg & & $\mathrm{im}$ & \\
\hline & & orf & & 8 & \\
\hline & & & & Irg & \\
\hline nohen & & 3. & & - & \\
\hline & & .1 & & lausen & \\
\hline & & 1 & & $\mathrm{n}$ & \\
\hline & & $\operatorname{sen}$ & & hausen & \\
\hline den & & & & 14 & \\
\hline ausen & & $\operatorname{urg}$ & & gen & \\
\hline 1 & & 1] & & . & \\
\hline dorf & & J & & 50.1 & \\
\hline & & hen & & 51. & \\
\hline & & ichen & & . & \\
\hline & & & & & \\
\hline 1 & & . 1 & & Niederb.) & \\
\hline h & & asen & & nen ... & \\
\hline ausen . . & & n & & dorf & \\
\hline nchen & & & & & \\
\hline & & & & & \\
\hline en & & & & & \\
\hline & & - & & rth & \\
\hline 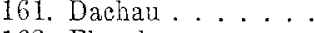 & & & & lünchen & \\
\hline & & D & & 162.1 & \\
\hline rth & & & & - & \\
\hline & & & & & \\
\hline & & den & & $\mathrm{ch}$ & \\
\hline 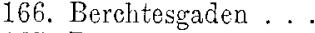 & & rth & 60 & 166. Berchtesgaden & \\
\hline O1. Dez-Alnt Augsourg & & 167. Bez-Amt Augsburg & .0000 & 167. Bez-Amt Augsburg & \\
\hline
\end{tabular}

Die Zahlen der Gesamtsterblichkeit vou Männern und Frauen der ersten senkrechten Reihe der vorhergehenden Tabelle wurden zur Herstellung der Karte benutzt. Sie weicht in beträchtichem Masse von der im .Jahre 1902 in der Zeitschrift für Hygiene, Bd. 40, veröffentlichten ab, wem sie auch im ganzen mit ihr übereinstimmt. Die Abweichung ist Folge der Verbesserung durch genauere Altersberïcksichtigung 
und richtige Verteilung der ausserhalb ihres Wohnortes Gestorbenen auf ihren Erkrankungsort.

Eine Linie, welche den Lauf der Donau angibt, lässt auf den ersten Blick erkennen, dass eine grössere Häufigkeit des Krebses sich in Bayern südlich der Donau findet und dass nördlich von ihr die Krebssterblichkeit eine geringere ist. Wührend diese, auf die bayerische Gesamthevölkerung iiber 30 Jahre als Standardbevölkerung berechnet, im Süden meist zwischen 2-3000 Todesfällen auf 1000000 Lebende schwankt, kommen von den 25 Bezirken mit über 3000 nur 3 auf den nördlichen Teil, und davon liegen 2, Nördlingen und Donauwörth, an der Grenze gegen Südbayern, und nur ein einziger, Miltenberg, ist ganz ron ihm getrennt. Anderseits kommt von den 37 Bezirksämtern mit einer Krebssterblichkeit von weniger als 2000 Todesfällen kein einziges auf Südbayern, wenn man bei den 4 nur teilweise dazugehörigen Bezirksämtern Stranbing, Deggendorf, Vilshofen und Passau, welche teils südlich, teils nördlich der Donau liegen, die Sterblichkeit für ihre durch die Donau getrennten Teile gesondert berechnet.

Um grosse Zahlen zu gewinnen und örtliche Zufälligkeiten auszuscheiden, ist es nunmehr nötig, die Zahlen der einzelnen Regierungsbezirke zusammenzustellen und am besten aus diesen drei grosse Gruppen zu bilden:

\section{A. Südbayern.}

a) Oberbayern, welches mit Ausuahme eines Teiles des Bezirlssamts Ingolstadt ganz südlich der Donau liegt.

b) Niederbayern südlich der Donau (nur vom Bezirksamt Kelheim wurde der nördlich der Donau liegende Teil`mitgerechnet).

c) Schwaben, das mit 21/2 Bezirksämtern noch über die Donau himübergreift (Nördlingen, Donauwörth und ein Teil von Dillingen).

\section{B. Mittelbayern.}

a) Niederbayern nördlich der Donau.

b) Oberpfalz.

c) Mittelfranken.

\section{c. Nordbayern.}

a) Oberfranken.

b) Unterfranken.

c) Pfalz.

Von diesen grossen Gruppen wurde im Folgenden, um schärfere Unterschiede zu gewinnen, nur die erste und dritte miteinander verglichen, nicht anch die zweite. Diese bildet natürlicherweise einen Uebergang, mamentlich auch in bezug auf Stammesverschiedenheit. Ihre Bevölkerung ist $z . T$. bayexisch (in Niederbayern und vorwiegend in der Oberpfalz), z. T. fränkisch (besonders in Mittelfranken), während Südbayern nur Bayern und Schwaben, Nordbayern nur Franken zu Bewohnern hat. 
Es ist dabei aber gleich zu betonen, dass daher rührende Unterschiede der Krebshäufigkeit nicht durch Rasseverschiedenheit, worüber später gesprochen wird, sondern nach meiner Ansicht nur durch die verschiedéne Lebensweise der Stämme veranlasst werden. Ausserdem sprach noch ein anderer Grund dafür, von Vergleichungen mit Mittelbayern abzusehen, welcher ebenfalls später erörtert werden soll.

Statt der Grundzahlen werden in den folgenden Tabellen nur die Sterblichkeitszahlen für alle Krebsfälle und die einzelnen Lokalisationen

Tabelle IV a.

Krebssterblichkeit der Männer.

\begin{tabular}{|c|c|c|c|c|c|c|c|c|c|}
\hline & \multicolumn{4}{|c|}{ Alle Krebsfälle } & \multirow{2}{*}{ 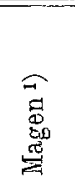 } & \multirow[b]{2}{*}{ 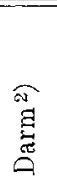 } & \multirow{2}{*}{ 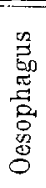 } & \multirow{2}{*}{$\mid \begin{array}{c}0 \\
0 \\
0 \\
0 \\
0 \\
0 \\
0.0 \\
0 \\
0 \\
0\end{array}$} & \multirow[b]{2}{*}{$\begin{array}{l}\widehat{\pi} \\
0 \\
2 \\
0 \\
3 \\
3\end{array}$} \\
\hline & $\begin{array}{c}30-50 \\
\text { Jahre }\end{array}$ & $\begin{array}{c}50-70 \\
\text { Jahre }\end{array}$ & $\begin{array}{c}\text { über } 70 \\
\text { Jahre }\end{array}$ & $\begin{array}{c}\text { über } 30 \\
\text { Jabre }\end{array}$ & & & & & \\
\hline Oberbayern & 393,37 & 1593,74 & 707,77 & 2694,88 & 1638 & 403 & - & - & - \\
\hline $\begin{array}{l}\text { Niederbayern } \cdot . \cdot 5 \\
\left.\text { [siddlich der Donau }{ }^{5}\right)\end{array}$ & 314,68 & 1263,96 & 552,75 & 2131,39 & 1442 & 182 & - & - & - \\
\hline Schwaben ..... & 389,67 & 1796,37 & 719,13 & 2905,17 & 1932 & 338 & - & - & $=$ \\
\hline Sädbajorn. & 382,29 & 1578,21 & 661,20 & 2621,70 & 1666 & 347 & 87 & 60 & 75 \\
\hline $\begin{array}{l}\text { Niederbayern ..... } \\
\text { (nördlich der ponau) }\end{array}$ & 232,18 & 860,43 & 341,84 & 1434,45 & 883 & 157 & - & - & - \\
\hline $\begin{array}{l}\text { Oberpfalz . . . . } \\
\text { Mittelfranken }\end{array}$ & $\begin{array}{l}314,57 \\
325,65\end{array}$ & 1354,65 & 428,73 & 2097,95 & 1501 & 239 & - & - & - \\
\hline Mittelbayern . & 312,72 & 1289,65 & 472,52 & 2074,89 & 1356 & 271 & 90 & -1 & 56 \\
\hline
\end{tabular}

\begin{tabular}{l|l|l|l|l|l|l|l|l|l|l}
\hline Pfalz ........ . & 221,00 & 1264,59 & 636,55 & 2122,15 & 1257 & 165 & - & - & - \\
Oberfranken . . . & 256,70 & 1227,81 & 408,51 & 1893,02 & 1343 & 174 & - & - & - \\
Unterfranken ... & 285,64 & 1279,50 & 552,62 & 2117,76 & 1363 & 227 & - & - & - \\
\hline Nordbayern ... . & 251,16 & 1258,89 & 507,07 & 2017,12 & 1317 & 182 & 127 & 32 & 60 \\
\hline Bayer . . . . . . & 321,95 & 1400,31 & 561,80 & 2284,15 & 1473 & 245 & 105 & 45 & 63
\end{tabular}

1) Begreift Magen, Leber, Gallengänge, Pankreas, Milz.

2) Darm und Rektum.

3) Bauchfell, Netz, "Unterleib", „im Becken", Baucheingeweide, Mesenterialund Retroperitonealdrüsen der Angaben der Leichenschauscheine, also jedenfalls viele unsichere Diagnosen. "Peritoneum" wurde besonders berechnet, um einen Masstab zu geben für die Genauigkeit der Diagnose, dann für die Zahl der Fälle, die hauptsächlich den Verdauungs- und weiblichen Geschlechtsorganen zuzurechnen wären.

4) Lippen, Zunge, Pharynx, Keblkopf.

5) Begreift hier auch die nördlich der Donau gelegenen Teile der Bezirksämter Straubing, Deggendorf, Vilshofen und Passau. 
Tabelle IVb.

Krebssterblichkeit der Frauen.

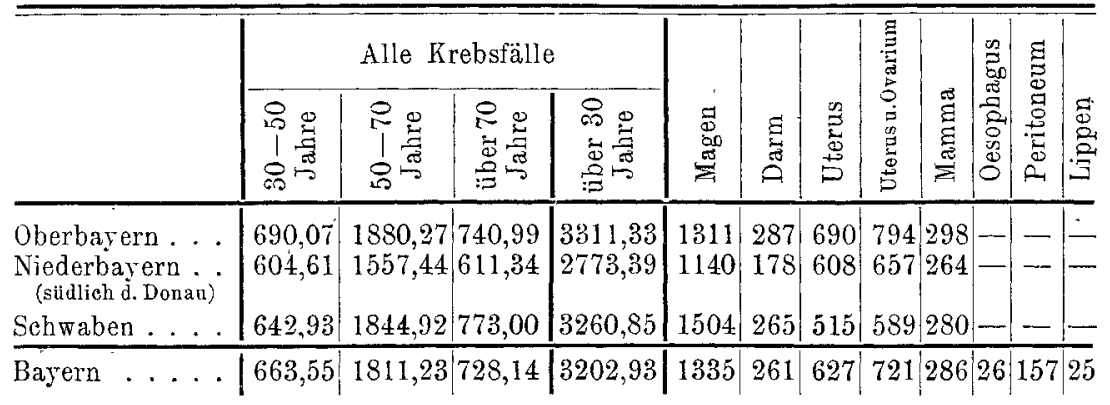

\begin{tabular}{c|c|c|c|c|c|c|c|c|c|c|c|c|c}
\hline $\begin{array}{c}\text { Niederbayern . . } \\
\text { (nördl. d. Donau) }\end{array}$ & 563,37 & 925,80 & 366,16 & 1855,33 & 740 & 124 & 396 & 438 & 177 & - & - & - \\
Oberpfalz . . . & 470,26 & 1356,01 & 465,44 & 2291,71 & 1102 & 215 & 328 & 362 & 201 & - & - & - \\
Mittelfranken . & 542,15 & 1538,43 & 640,83 & 2721,41 & 1194 & 255 & 502 & 559 & 200 & - & - & - \\
\hline Mittelbayern . . & 521,53 & 1393,70 & 534,28 & 2449,61 & 1097 & 219 & 428 & 487 & 195 & 32 & 118 & 18
\end{tabular}

\begin{tabular}{l|l|l|l|l|l|l|l|l|l|l|l|l|l|l}
\hline Pfalz. . . . . & 365,36 & 1454,39 & 647,62 & 2467,36 & 1371 & 190 & 243 & 286 & 118 & - & - & - \\
Obcrfranken. . & 423,70 & 1263,63 & 427,61 & 2114,94 & 1012 & 179 & 252 & 285 & 152 & - & - & - \\
Unterfranken . & 434,90 & 1435,05 & 581,70 & 2451,66 & 1338 & 235 & 331 & 372 & 217 & $-\ldots$ & - & - \\
\hline Nordbayern . . & 404,26 & 1390,03 & 555,53 & 2349,82 & 1241 & 200 & 272 & 311 & 159 & 37 & 93 & 19 \\
\hline Bayern $\ldots . .$. & 542,74 & 1566,45 & 623,54 & 2732,74 & 1244 & 233 & 460 & 521 & 222 & 30 & 126 & 21
\end{tabular}

fïr die einzelnen Regierungsbezirke, einzelne weniger wichtige Lokalisationen nur für jede der drei Gruppen angegeben ${ }^{1}$ ).

Eine graphische Darstellung des Wichtigsten dieser Tabelle gibt Tafel III.

Wir sehen aus der Tabelle ferner, Reihe $1-3$, dass die grössten Unterschiede der allgemeinen Krebssterblichkeit zwischen Süd- und Nordbayern namentlich in den jüngeren Altersklassen von 30-50 Jahren vorkommen. In diesen haben die Männer eine Uebersterblichkeit von $152 \mathrm{pCt}$., in den folgenden zwei Altersklassen nur von 125 und $130 \mathrm{pCt}$. gegen $100 \mathrm{im}$ Norden, und auch bei den Frauen ist das Verhältnis entsprechend 164 , später 130 und 131 pCt. Die verhältnismässige Senkung

1) Von den Grundzahlen werden im nächsten Hefte der Zeitschrift des Kgl. Bayerischen Statistischen Landesamts namentlich a) eine Tabelle über das Vorkommen aller einzelnen Organlokalisationen in den Bezirksämtern und Städten des Königreichs, und b) eine Tabelle mit Angabe der Lokalisationen für die $10 j a ̈ h r i g e n A]$ tersklassen, gesondert nach den 8 Regierungsbezirken, veröffentlicht werden. 
Tabelle V.

Uebersterblichkeit in Südbayern in den verschiedenen Altersklassen.

\begin{tabular}{|c|c|c|c|c|c|c|c|c|}
\hline & \multicolumn{4}{|c|}{ Männer } & \multicolumn{4}{|c|}{ Frauen } \\
\hline & $\begin{array}{l}0 \\
0 \\
0 \\
0\end{array}$ & 옹 & 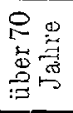 & 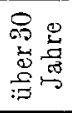 & $\begin{array}{l}0 \\
0 \\
10 \\
0.5 \\
0\end{array}$ & $\stackrel{8}{1}$ & 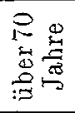 & 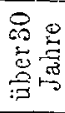 \\
\hline Alle Krrebsfïlle & 152 & 125 & 130 & 130 & 160 & 130 & 131 & 136 \\
\hline Magen, Leber usw. . & 148 & 119 & 135 & 127 & 109 & 103 & 119 & 108 \\
\hline Darm und Rektum . & 167 & 198 & 185 & 190 & 137 & 126 & 137 & 131 \\
\hline Oesophagus ..... & 89 & 73 & 49 & 88 & 100 & 67 & 72 & $8 s$ \\
\hline Peritoneum. & 176 & 256 & 122 & 138 & 171 & 169 & 162 & 168 \\
\hline Lippen usw. . & 133 & 115 & 149 & 119 & 71 & 131 & 189 & 133 \\
\hline Uterus . . . . & - & - & 一 & - & 268 & 215 & 198 & 220 \\
\hline Uterus und Ovarien & - & 一 & 一 & 一 & 272 & 218 & 196 & 232 \\
\hline 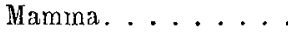 & - & - & - & - & 138 & $196^{\circ}$ & 201 & 180 \\
\hline
\end{tabular}

in der Lebensperiode zwischen dem 50. und 70. Jahre scheint, wie ans Tabelle $\mathrm{V}$ hervorgeht, besonders durch die verhälnismässig geringere Erkrankung an Magenkrebs in Sïdbayern veranlasst zu sein.

Die Uebersterblichkeit des Südens nimmt bei den Männern nach dem 50. Jahre für Darm und Rektum noch ansehnlich zu, bei den Franen für die Mamma; dagegen bei den Männern für den Oesophagus und bei den Frauen für die inneren Genitalien ab. Wenn man aber die Zahlen für alle Lokalisationen über dem 30. Jahre betrachtet, so erhält man doch im ganzen den Eindruck, als ob die Krebsagentien in den schwächer befallenen Landesteilen erst im höheren Lebensalter die Krelsshäufigkeit derjenigen der stärker befallenen Landesteile annäherten, sei es, dass die Reize erst später die nötige Häufung zur Erzeugnng der Krankheit erreichen, sei es, dass die nötige Disposition erst später eintritt, oder sei es endlich, dass in den stark befallenen Teilen durch frühen Tod viele Krebsdisponierte für die späteren Alter wegfallen.

Unter den einzelnen Lokalisationen ist die Uebersterblichkeit im Süden am grössten bei den Männern für Darm und Rektum: 190 pCt., bei den Frauen für Uterus 220, Uterus und Ovarien 232, Mamma $180 \mathrm{pCt}$. Gegenüber der Uebersterblichkeit bei allen anderen angeführten Lokalisationen ist höchst auffällig eine Mindersterblichkeit im Süden für den Oesophagus, bei den Männern 6s, bei den Fratuen $88 \mathrm{pCt}$. der nordbayerischen. (Siehe Tafel IV.)

Die Häufigkeit des Krebses muss für Stadt und Land gesondert untersucht werden, schon wegen der früher erwähnten Verschiedenheit der Erhebungen. Die folgende Tabelle gibt die Sterblichkeitsziffern für die drei Gruppen von Regierungsbezirken getrennt für Stadt und Land. 
Tabelle VI.

Krebssterblichkeit in Stadt und Land.

a) Mäinner.

\begin{tabular}{|c|c|c|c|c|c|c|}
\hline & Alle Fälle & $\begin{array}{l}\text { Magen } \\
\text { usw. }\end{array}$ & Darm & Rektum & $\begin{array}{l}\text { Oeso- } \\
\text { phagus }\end{array}$ & $\begin{array}{l}\text { Peri- } \\
\text { toneum }\end{array}$ \\
\hline $\begin{array}{l}\text { Suidbayern ... } \\
\text { Mittelbayern . . } \\
\text { Nordbayern. . }\end{array}$ & $\begin{array}{l}2883 \\
2563 \\
2314 \\
\end{array}$ & $\begin{array}{l}1474 \\
1425 \\
1287 \\
\end{array}$ & $\begin{array}{l}263 \\
245 \\
188 \\
\end{array}$ & $\begin{array}{l}300 \\
298 \\
195 \\
\end{array}$ & $\begin{array}{l}122 \\
139 \\
165 \\
\end{array}$ & $\begin{array}{l}90 \\
53 \\
62 \\
\end{array}$ \\
\hline Städte .... & 2697 & 1428 & 236 & 283 & 134 & 74 \\
\hline $\begin{array}{l}\text { Südbayern . . } \\
\text { Mittelbayern . . } \\
\text { Nordbayern . . }\end{array}$ & $\begin{array}{l}2523 \\
1934 \\
1953 \\
\end{array}$ & $\begin{array}{l}1757 \\
1346 \\
1366 \\
\end{array}$ & $\begin{array}{r}139 \\
103 \\
91 \\
\end{array}$ & $\begin{array}{r}120 \\
86 \\
91\end{array}$ & $\begin{array}{l}91 \\
79 \\
81\end{array}$ & $\begin{array}{l}52 \\
26 \\
27\end{array}$ \\
\hline Land ..... & 2203 & 1536 & 115 & 104 & 82 & 38 \\
\hline
\end{tabular}

b) Frauen.

\begin{tabular}{l|c|c|c|c|c|c|c|c|c}
\hline & $\begin{array}{c}\text { Alle } \\
\text { Fälle }\end{array}$ & $\begin{array}{c}\text { Magen } \\
\text { usw. }\end{array}$ & Darm & $\begin{array}{c}\text { Rek- } \\
\text { tum }\end{array}$ & $\begin{array}{c}\text { Oeso- } \\
\text { phagus }\end{array}$ & $\begin{array}{c}\text { Peri- } \\
\text { toneum }\end{array}$ & Uterus & $\begin{array}{c}\text { Uterus } \\
\text { und } \\
\text { Ovarien }\end{array}$ & Mamma \\
\hline Südbayern .. & 3514 & 1321 & 197 & 145 & 30 & 195 & 759 & 837 & 293 \\
Mittelbayern . & 3083 & 1254 & 211 & 144 & 43 & 182 & 602 & 673 & 251 \\
Nordbayern... & 2649 & 1159 & 196 & 88 & 44 & 169 & 355 & 402 & 229 \\
\hline Stälte...... & 3250 & 1286 & 200 & 136 & 36 & 187 & 649 & 728 & 270 \\
\hline Südbayern . . & 3035 & 1521 & 143 & 75 & 24 & 137 & 552 & 612 & 284 \\
Nittelbayern . & 2184 & 1132 & 108 & 54 & 27 & 89 & 346 & 383 & 173 \\
Nordbayern.. & 2195 & 1243 & 118 & 70 & 29 & 81 & $\mathbf{2 5 2}$ & 303 & 172 \\
\hline Land ..... & 2563 & 1311 & 126 & 68 & 27 & 108 & 417 & 464 & 222
\end{tabular}

Die rerschiedene Gesamtsterblichkeit von Stadt und Land wurde schon früher besprochen und es wurde ausgeführt, dass die Sterblichkeit in den Städten meistens grösser, aber im Regierungsbezirke Schwaben, wenn auch nicht nach den vorliegenden Angaben, doch wahrscheinlich tatsächlich auf dem Lande höher ist.

Früher hatte man angenommen, dass die Sterblichkeit allgemein in den Städten viel bedeutender sei. Es trifft dies aber keineswegs überall zu. Ein Ueberwiegen der ländlichen Krebssterblichkeit wurde schon von Robertson (14) bei Ausscheiden der Ortsfremden für Schottland und von de Bovis (15) für Rotterdam angegeben. Es scheinen allerdings diese Berechnungen ohne Altersberücksichtigung vorgenommen worden zu sein und sind deshalb nicht zu sicheren Schlïssen geeignet. Auch bei den Untersuchungen von Geirsvold (16), welcher für Norwegen zum gleichen Ergebnisse kommt, wurde jener Fehler nicht rermieden. Die Uebersterblichkeit des Landes ist aber hier so gross, 816 gegen 519 Todesfälle auf 
die Million Lebender, dass sie auch bei Berïcksichtigung der wohl zahlreicheren alten Lente auf dem Lande als wirklich bestehend angenommen werden kann. Es wird sonach auch der Schluss, welchen ich vor Jahren gezogen habe, noch heute richtig sein, dass der Krebs nicht nur anscheinend, sondern tatsächlich im allgemeinen in den Städten häufiger ist als auf dem Lande, „dass aber jedenfalls auch nicht selten das entgegengesetzte Verhältnis besteht". Es ist ja auch von vornherein zu vermuten, dass das Gesamtprodukt der verschiedenen Koeffizienten, welche die Häufigkeit des Krebses beeinflussen und welche spezifisch städtische und spezifisch ländliche Einwirkungen in sich begreifen, je nach ihrer Kombination eine höhere Sterblichkeit bald in der Stadt, bald auf dem Lande ist.

Auf Seite 204 wurde schon eine Vergleichung der Sterblichkeit auf dem Lande mit der städtischen in ganz Bayern mitgeteilt; die folgende Tabelle gibt einen Vergleich für unsere drei bayerischen Gruppen, auch für die einzelnen Lokalisationen.

Tabelle VII.

Sterblichkeit auf dem Lande in Prozenten der städtischen.

\begin{tabular}{|c|c|c|c|c|c|c|c|c|}
\hline & \multicolumn{4}{|c|}{ Männer } & \multicolumn{4}{|c|}{ Frauen } \\
\hline & 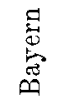 & 富高 & 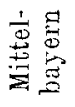 & 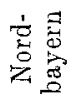 & 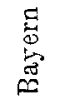 & 涪密 & 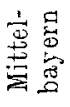 & 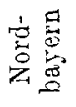 \\
\hline Alle Fülle. & 82 & 88 & 76 & 85 & 79 & 86 & 73 & 83 \\
\hline Magen usw. ..... & 104 & 119 & 94 & 106 & 102 & 115 & 90 & 107 \\
\hline 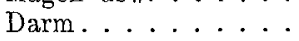 & 49 & 53 & 42 & 48 & 63 & 73 & 51 & 60 \\
\hline Rektum ..... & 37 & 40 & 29 & 47 & 50 & .52 & 37 & 79 \\
\hline Oesophagus $\ldots \ldots$ & 61 & 74 & 57 & 49 & 73 & 82 & 63 & 66 \\
\hline Peritoneum ..... & 52 & 58 & 50 & 43 & 58 & 70 & 49 & 48 \\
\hline Uterus . . . . . . & - & - & - & - & 64 & 73 & 57 & 71 \\
\hline Uterus und Ovarien . & - & - & - & - & 63 & 69 & 57 & 75 \\
\hline Mamma ....... & - & - & - & - & 82 & 97 & 69 & 74 \\
\hline
\end{tabular}

Diese Zusammenstellung erlaubt zunächst die Vertrauenswürdigkeit der ländlichen Angaben einigermassen zu prüfen. Wenn man davon ausgeht, dass die geringere Sterblichkeit auf dem Lande zu gutem Teil Folge mangelhafter Diagnosen ist, so sollte man eine besonders grosse Differenz bei den schwer diagnostizierbaren Krebsen erwarten.

Stellt man nun unsere Hauptgruppen nach ihrer Diagnostizierbarkeit von den leichter zu den schwerer diagnostizierbaren zusammen (ähnlich der Einteilung des Englischen Krebskomitees), so erhält man die Folge: 1. Namma, 2. Rektum, 3. Uterus, 4. Oesophagns, 5. Magen usw., 6. Darm, 7. Peritoneum. 
Reiht man sie nach ihrer relativen Häufigkeit auf dem Lande gegenüber der Stadt, ausgehend von der grössten Häufigkeit, so orduen sie sich:

1. Magen 102-104, 2. Mamma 82, 3. Oesophagus 61-73, 4. Uterus 64, 5. Peritoneum 52-58, 6. Darm 49-63, 7. Rektum 37-50.

Die beiden Reihen entsprechen sich nur beim Darm, annähernd bei Mamma, Oesophagus und Uterus, gar nicht bei Rektum und Magen. Man kann darans vermuten, dass die Diagnose auf dem Lande nicht wesentlich unrichtiger ist als in der Stadt, - abgesehen davon, dass auf dem Lande mehr nichtbehandelte Fälle vorkommen und deshalb für die Krebsdiagnose ausfallen.

Es spricht dafïrr auch, dass unter den gemeldeten Krebsfällen die Angabe des Sitzes der Krankheit auf dem Lande nicht häufiger mangelt als in der Stadt. Diese Fälle betragen im ganzen Lande für die Männer 1,9. für die Frauen 2,2 pCt., aber in der Stadt München für erstere 3,1, für letztere 2,2 pCt. Die Unterlassung der Angabe des Sitzes findet sich merkwürdigerweise überall vor allem bei der Diagnose Sarkom. Dagegen wird allerdings die grössere Uebersterblichkeit des Südens an Krrebs des Peritoneum (Tabelle VIII) zum Teil von ungenaueren Diagnosen herrühren.

Zieht man auch in Betracht, dass immerhin ein Teil der Untersterblichkeit auf dem Lande nur eine scheinbare, aus unvollständiger Erhebung hervorgehende ist, so möchte man doch schliessen, dass der Krrebs von Darm und Rektum, dann der weiblichen Genitalien auf dem Lande seltener ist, während der Magen- und Leberkrebs hier sogar häufiger vorkommt. Die Todesfälle an Darmkrebs betragen auf dem Lande nur 42-53 pCt. der städtischen bei den Männern, $51-73 \mathrm{pCt}$. bei den Frauen; diejenigen an Rektumkrebs 29-47, bzw. 37-79 pCt. Diese Unterschiede sind so gross, dass sie kaum durch unvollständigere Erhebung auf dem Lande allein zu erklären sein dürften, besonders wenn man berücksichtigt, dass der Rektumkrebs wenigstens im Verlaufe der Krankheit doch meist zu den leichter diagnostizierbaren gehört, wie ihn auch das Englische Komitee zu den "intermediate" rechnet. Zur Erklärung dieser Unterschiede der Häufigkeit wäre bei beiden Lokalisationen zunächst an den Einfluss verschiedener Ernährung und der sitzenden Lebensweise in der Stadt zu denken. Auch der grosse Unterschied bei Krebs des Uterus und der'Ovarien dürfte nicht nur ein scheinbarer sein; auch für sein häufigeres Vorkommen in der Stadt dürfte die städtische Lebensweise mit ihren mannigfachen gesundheitswidrigen Einflüssen verantwortlich zu. machen sein.

Betrachten wir endlich noch, wie sich getrennt für Stadt und Land die Krebssterblichkeit in Südbayern zu der in Nordbayern verhält. 
Tabelle VIII.

Sterblichkeit von Südbayero in Prozenten von Nordbayern.

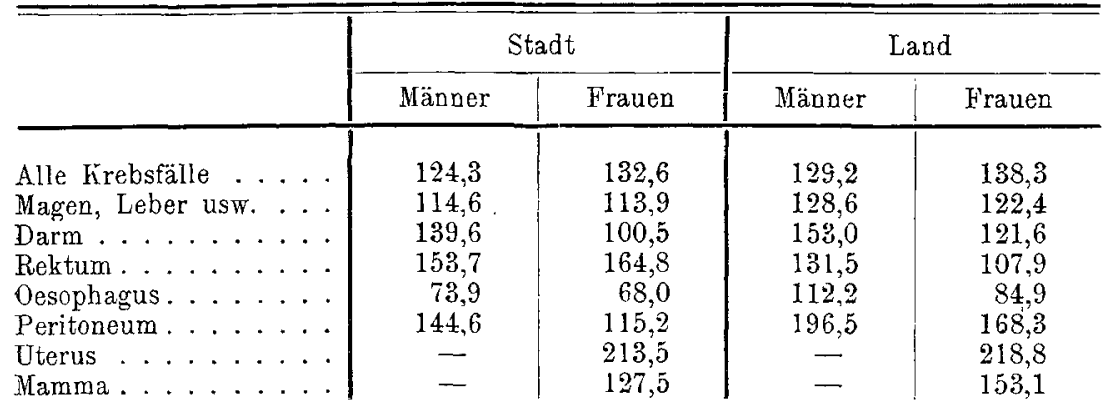

Die allgemeine Uebersterblichkeit ist im Süden, wie früher schon bemerkt wurde, auf dem Lande noch grösser als in der Stadt. Bei den einzelnen Lokalisationen trifft dies namentlich beim Magenkrebs zu; ja bei dem Oesophaguskrebs steigt die Häufigkeit auf dem Lande bei den Männern so sehr, dass sie sogar diejenige im Norden übertrifft. Rechnet man Darm und Rektum zusammen, so ist umgekehrt die Uebersterblichkeit des Südens in den Städten grösser, besonders bei den Frauen, was auch für die obige Annahme der ätiologisehen Bedeutung der sitzenden Lebensweise sprechen dürfte. Bei den weiblichen Genitalien einschliesslich der Mamma ist dagegen wieder eine grössere Uebersterblichkeit des Südens auf dem Lande vorhanden.

Bezüglich der beiden Geschlechter mögen nur folgende Zahlen angeführt werden, da die Unterschiede in den drei Gruppen im ganzen wenig bedeutend sind.

Die weibliche Krebssterblichkeit ist im Verhältnis zur männlichen $(=100)$ :

\begin{tabular}{ll|c|c|c}
\hline & & $\begin{array}{c}\text { bei allea } \\
\text { frebsfällen }\end{array}$ & $\begin{array}{c}\text { bei allen Fällen, } \\
\text { ohne die der weib- } \\
\text { lichen Genitalien }\end{array}$ & $\begin{array}{c}\text { bei } \\
\text { Magenkrebs }\end{array}$ \\
\hline in Südbayern & & & &
\end{tabular}

II.

Es liegen nunmehr seit zwei, teilweise seit über drei Jahrzehnten ausser den staatlichen Sanitätsberichten der süddeutschen Staaten (17), der Schweiz (18) und Oesterreichs (19) ausgedehnte Privatarbeiten, so ron Nencki (20), mir (21), Rosenfeld (22), Prinzing (23), Köhl (24), 
Werner (25) für jenes Gebiet grosser Krebssterblichkeit vor, welches ich zuerst in seiner ganzen Ausdehnung von Wien bis Genf nachgewiesen habe, und in jeder neuen Arbeit wird bestätigt, dass in seinem Bereich die Krebssterblichkeit höher ist, als in den umgebenden Ländern. Auch heute noch gilt, was Prinzing vor 10 Jahren für die Landesteile Oesterreichs sagte: „Der Krebs fordert in den Gebieten, bezüglich deren er früher am häufigsten war, auch heute noch die zahlreichsten Opfer", und zwar gilt dies nicht nur für Oesterreich, sondern auch für Süddeutschland und die Schweiz. Wenn auch ein Teil dieser zahlreichen Untersuchungen nicht mit Berücksichtigung des Altersaufbaues gemacht wurde, so geschah dies doch in den meisten Fällen. Die vorliegende Untersuchung berücksichtigt ihn in weitgehendem Grade durch Benutzung einer Standardbevölkerung als Massstab, und sie hat, wie früher bemerkt, zum ersten Mal für Bayern die gestorbenen Ortsfremden ihren betreffenden Wohnorten zugerechnet. Von den zwei einzig noch möglichen Fehlerquellen, der nicht ganz gleichmässigen Erhebung und dem Einfluss der in den einzelnen Landesteilen verschieden vertretenen städtischen Bevölkerung, wurde letztere als völlig unwesentlich nachgewiesen und für unsere Hauptvergleichung zwischen Süd- und Nordbayern ist sie ganz belanglos, während die erstere dafür überhaupt wegfällt.

Somit liegt gar kein stichhaltiger Grund mehr ror, an der Verschiedenheit der Häufigkeit des Krebses in den einzelnen Teilen Bayerns, namentlich Südbayerns einer- und Nordbayerns andererseits zu zweifeln. Es handelt sich nicht mehr darum, zu erörtern, $o b$, sondern warum diese Unterschiede bestehen.

Es ist ein Verdienst der Statistik, dass sie die vielen Einflüsse, welche man manchmal mit grosser Einbildungskraft, aber unzureichender Begründung mit der Entstehung der Krankheit in Zusammenhang gebracht hat, schon einigermassen sichten konnte. Doch soll sie sich nicht nur die Aufgabe stellen, qualitativ die Bedingungen der Krankheit herauszufinden (diese Aufgabe, noch vor kurzem selbst von Medizinalstatistikern als unmöglich erklärt, wird ihr jetzt allgemein zugestanden), sondern sie soll sich neben qualitativen auch quantitative Ziele setzen, sie soll auch darauf ausgehen, für die rerschieden gearteten Koeffizienten auch ein gewisses Mass zu finden, - soweit dies möglich ist. Ich verkenne die Schwierigkeiten nicht. Selbstverständlich kann dieses Mass aus verschiedenen Gründen nicht entfernt ein absolutes, immer giltiges sein. Die Medizinalstatistik hat gerade in der komplizierten Krebsätiologie nicht gleichbleibende Grössen zur Verfügung, sondern der Komplex der selbst veränderlichen Grössen der einzelnen Koeffizienten zeigt nach Ort und Zeit wechselnde Kombinationen. Die Massbestimmung der einzelnen "Bedingung" kann daher nur immer für ein bestimmtes Land und eine 
bestimmte Zeit annähernd richtig sein; für andere Zeiten und Orte gilt sie nur als bestmöglicher Masstab, was aber immer noch besser ist als gar kein Masstab oder allenfalls die Abschätzung von Haupt- und Nebenursachen.

Ueberblicken wir in aller Kürze die bisher als ätiologische Agentien aufgestellten Einflüsse, namentlich soweit sie für die verschiedene örtliche Häufigkeit des Krebses in Betracht kommen könnten. Der hervorragende Einfluss des Alters und auch der des Stadt-oder Landlebens ist nach unserer Methode ausgeschieden. Es ist auch nicht nötig, hier auf individuell wirkende Ursachen, wie Traumen, Lupus, vorhergegangene Syphilis oder Geburten einzugehen. Für manche der aufgestellten. Ursachen wurden überhaupt bis jetzt keine endgiltigen Beweise beigebracht, so für das Klima, soweit es wenigstens direkt einwirken sollte, einschliesslich des wechselnden Gehalts von Luft, Boden und Wasser an Radium (26), ebenso für die Wohndichte, besonders nach den negativen Ergebnissen von Kiaer und Geirsvold (27), und die Wohlhabenheit, sofern letztere für die Gesamtsterblichkeit an Krebs in Betracht kommt. Während man früher dieser und unter anderem namentlich dem übertriebenen Fleischgenuss schädliche Wirkungen zuschrieb, kamen andere Untersuchungen mehrfach zu einem entgegengesetzten Resultat. Schon Aschoff hatte für Berlin eine geringere Sterblichkeit bei den Almosenempfängern gefunden (2S), und nach den Mitteilungen des Bremischen. statistischen Amts (29) war bei den ärmeren Klassen die Sterblichkeit auch geringer als bei den wohlhabenderen. Die Untersuchungen A schoffs sind aber olne genamere Berücksichtigung des Alters und mit einer unrichtigen Methode angestellt, und nach Rosenfeld konnte weder in Wien, noch in Berlin, Stuttgart oder Hamburg ein Einfluss von Wohlhabenheit statistisch nachgewiesen werden. Dagegen dürfte die Wohlhabenheit für die Häufigkeit einzelner Lokalisationen von Bedeutung sein. Vor allem sind hier die bekannten Untersuchungen von Weinberg und Gastpar (30) anzuführen, nach denen bei Altersberüicksichtigung sich eine bedeutend höhere Sterblichkeit an Uteruskrebs bei den ärmeren Klassen in Stuttgart herausstellte. Weinberg will aber, auch für die Häufigkeit der anderen Lokalisationen bei den ärmeren Klassen, nur provisorische Schlüsse zulassen; die Zahlen sprächen jedoch gegen das Vorherrschen des Krebses. in den höheren Klassen.

Damit sind wir zugleich zur Besprechung der Kultur, eines allerdings vieldeutigen Wortes übergegangen. Auch für sie ist liein tiefgreifender Einfluss festgestellt, denn die Behauptung Rosenfelds, dass wir in Gegenden mit höherer Kultur häufiger auf Krebs stossen, scheint mir nicht bewiesen, wenn wir von dem schon besprochenen komplizierten Verhältnis von Stadt und Land absehen. Ich erinnere an das gegenteilige Vorkommen, 
an das Maximum der Krebssterblichkeit in der Zentralschweiz, in Salzburg und Berchtesgaden, im bayerischen Schwaben, im württembergischen Donaukreis, im badischen Oberland, und andererseits an unsere Grosstädte Augsburg und Würzburg, welche geringere Krebssterblichkeit haben als die umliegenden Bezirksämter. Rosenfeld erklärt allerdings vorkommende Widersprüche durch die verschiedene Bedeutung, welche man dem Worte Kultur beilegt. Der Begriff Kultur, als ätiologischer Faktor aufgestellt, schliesst einerseits das Hasten und Jagen der modernen Gütererzeugung, andererseits den Missbrauch des Errungenen zur Verweichlichung, zum Wohlleben mit Hintansetzung gesundheitlicher Gebote ein. So entstehen die Nervosität (Julian Markuse), so die frühzeitige körperliche und geistige Abnutzung mit sittlicher Degeneration, wenn nicht direkt Krankheiten wie Syphilis, Alkoholismus. Diesen Gesamtbegriff mit seinen Folgen wird man gewiss nicht gerade in der Bevölkerung der genannten Landesteile mehr ausgeprägt finden als in ihrer Umgebung. So sehr man einen gewissen Einfluss der Kultur für möglich, ja für wahrscheinlich halten kann, so kann er jedenfalls nicht, so wenig wie die vorher besprochenen Einflüsse, als wesentliche Ursache für die gesamte Krebshäufigkeit angesehen werden, und in Bayern im besonderen sind es nicht die verkehr-und industriereichsten, wohlhabendsten und dichtestbewohnten Teile, welche am meisten an Krebs leiden (31).

Ueber den Beruf konnte ich nach den bayerischen Erfahrungen (32) das Ergebnis dahin zusammenfassen: Sicher scheint bis jetzt, dass nur spezifisch einwirkende Berufe einen entschiedenen ätiologischen Einfluss haben, wie der der Anilin-, Teer-, Paraffin-, Röntgenarbeiter, der Kaminfeger und der Bergleute von Schneeberg. Das letaterwähnte interessante Vorkommen wird meist auf die bearbeiteten Metalle, Kobalt und Arsen, von Ancke (33) auf die leicht lösliche Form des Kobaltarsens zurückgeführt. J. Colunheim (34) hat aber darauf hingewieseñ, dass in ähnlichen Bergwerken, z. B. im südlichen Schweden, die Krankheit unbekannt ist. Die Wahrscheinlichkeit läge vielmehr vor, dass es sich um eine Infektion dureh ein organisiertes Gift, welches sich vielleicht in den Grubenwassern findet, handle. Auch neuere Beobachtungen führen zu ähnlichen Annahmen. Härting Sohn (35) nimmt zwar die Einatmung giftiger Grubengase als Ursache an; doch sei auch eine infektiöse nicht ausgeschlossen; Weise (35) glaubt, dass die Sarkome auf lebende Schädlinge, vielleicht Schimmelpilze oder in deren Gemeinschaft lebende Wesen zurückzuführen sind. Es gäbe sogar in den Gruben Strecken, die bei den Arbeitern als besonders gefährlich gelten und Arnstein (36) sagt, dass die feuchtesten Schächte als die bedenklichsten gelten und die mit dem feuchten Holz hantierenden Zimmerleute besonders gefährdet sind. Welche Ursache hat die hohe Krebssterblichkeit, welche Payne für die Kupferbergleute in 
England gefunden hat? - Jedenfalls kann ein Einfluss des Berufes auf die Krebshäufigkeit ganzer Landesteile ausgeschlossen werden.

Auch ein auffallender Einfluss der Rasse wurde, etwa mit Ausnahme der jüdischen, noch nirgends in Europa nachgewiesen und dies ist um so begreiflicher, wenn ein Ausspruch Virchows (37) für unsere rassengemischte europäische Bevölkerung richtig ist, dass die Nationalitäten eigentlich gar nicht anthropologisch, sondern nur linguistisch zu behandeln sind. Selbst. für die Juden bleibt es fraglich, ob ihre meist geringer angegebene Sterblichkeit, wie für Krakau nach De Bovis (38), für Budapest und das Komitat. Bekes (39). Amsterdam (40), für die polnischen Juden in Nordamerika (39), auf die Rasse und nicht vielmehr auf ihre Lebenshaltung zu beziehen ist. Sogar die sehr unsicheren Behauptungen über die Seltenheit des Krebses bei unzivilisierten Völkern, namentlich der Tropen, enthalten dahingehende Angaben, dass z. B. die Neger von Sierra Leone (41) als Ureinwohner ziemlich frei von Krebs seien, aber nach Annahme europäischer Ernährung, Kleidung und Lebenshaltung zunehmend an Krebs erkrankten. Ich bin nach meinen Untersuchungen üher die Verhältnisse im Kanton Bern zu dem Resultat gekommen, dass in dem bearbeiteten Teil der Schweiz nicht der Unterschied der deutschen und französischen Rasse, sondern andere Einflüsse die verschiedene örtliche Häufigkeit des Krebses veranlassen. Für Bayern kann man um so weniger an einen Einfluss der Rasse denken, als es sich hier nur um Stammesunterschiede desselben Volkes handeln könnte. Selbst wenn diese "Stammesverschiedenheit" vielleicht weniger durch ursprïngliche Unterschiede als durch verschieden starke Vermischung: mit der früher im Lande wohnenden Bevölkerung verursacht sein sollte (J. Ranke), so ist sie für unsere Frage in Bayern wie in ganz Süddeutschland nicht von Belang.

Der Einfluss der Erblichkeit, welcher nach dafür sprechenden Erfahrungen beim Menschen wahrscheinlich gemacht worden war - ich nenne nur Young (43), Velpeau (44), Geirsvold (45), besonders aber Weinberg und Gastpar (46) -, ist num endlich bei Ratten und Mäusen unzweifelhaft nachgewiesen, so von Levin (47), Cuenot und Mercier (48), Bashford (49) und Slye (50). Soweit sie als Rasseeigenschaft von Bedeutung wäre, wurde sie schon gewürdigt; als individuelle Eigenschaft wäre sie nur infolge von Inzucht für die Topographie des Krebses zu berïcksichtigen. Vor einiger Zeit hat Jessup (51) die Behauptung aufgestellt, dass das Maximum der Krebshäufigkeit in der Schweiz sich in den geographisch oder durch religiöse Abschliessung isolierten Gebieten, wie Appenzell i. Rh., das Minimum an den Pässen und in den verkehrsreichsten Gegenden finde. Abgesehen daron, dass diese Aufstellung nicht mit anderen Tatsachen übereinstimmt (z. B. in der Schweiz selbst die grösste Häufigkeit in den Kantonen Schwyz, Zug und Luzern, eine sehr 
geringe im Berner Oberland vorkommt), gibt es in Bayern keine Gegenden, für welche die Inzucht von Bedeutung sein könnte. Es ist vielmehr nebenbei gesagt - zu befürchten, dass die stets zunehmenden Wanderungen der Bevölkerung immer mehr die topographischen Beziehungen des Krebses verwischen.

Alle bisher besprochenen Einwirkungen - um das Gesagte kurz zusammenzufassen - sind nicht geeignet, eine Erklärung für die verschiedene Krebshäufigkeit in Bayern zu geben. Sie konnten entweder bei der Berechnung vollständig ausgeschieden werden, wie Alter, Stadt und Land, oder sie können nur individuell wirken, wie Erblichkeit, in der Regel auch der Beruf, disponierende Krankheiten, wie Gallensteine, Magengeschwüre usw. Für die übrigbleibenden ist ein massgebender Einfluss auf ganze Bevölkerungen überhaupt noch nicht nachgewiesen, oder sie zeigen gerade in Bayern so geringe örtliche Unterschiede, dass sie nicht zur Erklärung gebraucht werden können. Dahin gehören Wohnungsdichte. industrielle Beschäftigung, Kultur, Wohlhabenheit, dann Klima, Rasseverschiedenheiten, Inzucht. Nach ihrer Verteilung in Bayern müsste man a priori sogar zum Teile eine ganz andere Verbreitung des Krebses im Lande erwarten.

Dagegen gibt es allerdings andere Faktoren, welche die Häufigkeit des Krebses bei Bevölkerungen grosser Landesteile oder selbst Länder beeinflussen können; es sind dies die Lebensgewohnheiten, wie Alkoholismus und Nichtstillen.

Betrachten wir zunächst die schädlichen Wirkungen des Alkohols. Schon seit Ende des 18. Jahrhunderts wurde die Entstehung des Magenkrebses auf chronischen Alkoholgenuss zurückgeführt. Dann wurde in den heute noch interessanten französischen Sammelforschungen, welche seit Beginn der 90 er Jahre besonders in der Normandie, dann in den Ardennen usw. angestellt wurden (52), zwar meist das schlechte Trinkwasser beschuldigt, doch auch von einzelnen Aerzten der starke Alkoholgenuss als ursächliche Schädlichkeit in Erwägung gezogen. In Deutschland hat meines Wissens zuerst Fircks (53), aber nach der fehlerhaften Methode der Beziehung der Krebstodesfälle auf 1000 überhaupt Gestorbene, eine Uebersterblichkeit des Krebses bei den mit alkoholischen Getränken Beschäitigten ausgerechnet. Später hat man vielfach die Häufigkeit des Krebses im obersten Teile der Verdauungsorgane bei Männern mit Reizungen in Verbindung gebracht [Redlich (54)]. In England hatte zuerst Payne (55) gefunden, dass nach Kaminfegern und Kupferbergleuten Brauer, Wirte und Wirtsbedienstete die höchste Krebssterblichkeit hatten. Die Berufsuntersuchungen von Tatham (56) für 1902-1905 hatten ein ganz entsprechendes Ergebnis: Nach den Kaminfegern mit 136 hatten die Gastwirtsbediensteten mit 133 die grösste Krebssterblichkeit gegenüber 86, 
welche alle Erwerbstätigen Londons als Durchschnitt hatten, und nach einer anderen Tabelle die Brauer 175 gegenüber 100 aller Berufe. Weder Payne noch Tatham dachten indes daran, die Schädlichkeit des Alkoholmissbrauchs auch zur Erklärung der verschiedenen örtlichen Häufigkeit des Krebses zu verwenden, wie denn überhaupt die Topographie des Krebses in neuerer Zeit ganz auffallend vernachlässigt wird.

Ich habe schon im Jahre 1906 (57) auf die ausserordentliche Häufigkeit des Oesophaguskrebses in einigen Teilen des Kantons Bern aufmerksam gemacht, dass in einem Bezirk auf 1000000 Gesamtbevölkerung jährlich 386 , in einem zweiten sogar 421 Todesfälle daran vorkommen und dass wallrscheinlich der dort stark rerbreitete Branntweingenuss dabei schuldig ist. Ich gebe zu, dass dies eine grobe Berechnungsweise gewesen ist; aber sie genügte in diesem Falle, wenn wir bedenken, dass in vielen Bezirken z. B. auch Süddeutschlands jene Sterblichkeit der Zahl sämtlicher Krebstodesfälle gleichkommt. Ich habe weiter bei den Untersuchungen über den Einfluss des Berufs auf die Krebssterblichkeit im 9. Bande dieser Zeitschrift diese für alle Erwerbstätigen über 30 Jahre zu 1419 auf 1000000 Lebende gefunden, für das Wirtsgewerbe: Wirte, Weinhändler, Brauer, Küfer aber zu 2132, also um die Hälfte höber. Ganz ähnliches Verhältnis zeigen die oben mitgeteilten Zahlen ron Payne und Tatham. Noch genauer ist eine spätere Berechnung für die folgenden Berufe unter Annahme rollkommen gleichen Altersaufbaues. Sie ergibt:

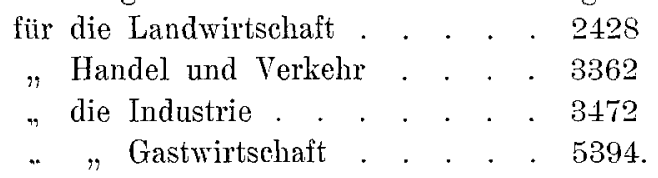

Das Verhältnis ist hier sogar noch ungünstiger für das Alkoholgewerbe, 155-222 pCt. Damit ist ein Massstal gefunden für die Schädlichkeit des Alkoholmisshrauchs. Man kann freilich nicht sagen, dass jene Uebersterblichkeit des Wirtsgewerbes allein dem Alkohol zuzuschreiben ist; man darf aber andererseits nicht vergessen, dass nicht alle Angehörigen jenes Gewerbes Alkoholisten sind. Ich betrachte rielmehr jenen Massstab, die Erhöhung der Krebssterblichkeit um die Hälfte durch den Alkoholmissbrauch für die Einzelindividuen eher noch als zu niedrig. Für ganze Bevölkerungen wird sich natürlich die Einwirkung des Alkohols nur schätzen, nicht berechnen lassen. Wenn man aber weiss, dass z. B. in Frankreich sich der Alkoholverbrauch von 1860 bis 1911 fast auf das Doppelte für jeden Kopf erhöhte, dagegen in Deutschland in den ersten 8 Monaten des Berichtsjahres 1910 der Branntweinverbrauch um iiber ein Viertel abgenommen hat, - ferner dass in Nürnberg der Bierkonsum ron 1890 bis 1908 von 344 auf 243 Liter zurüekgegangen ist, - andererseits, dass der Bierverbrauch in Bayern 1906 21/2 mal so 
gross war wie in Preussen und Sachsen, so kann kein Zweifel bestehen, dass die Folgen dieser Verschiedenheiten und Schwankungen sich auch in Unterschieden der Krebssterblichkeit zeigen müssen.

Ich habe ferner bei jenen Untersuchungen gefunden, dass bei Wirten insbesondere der Krebs des Darms und Rektum in Südbayern 2 mal häufiger vorkommt, als in Nordbayern und dagegen der Oesophaguskrebs im Norden 2 mal häufiger, als im Süden. Da nun in ähnlicher Weise bei der ganzen männlichen Bevölkerung (weniger bei den Frauen) im Süden der Krebs von Darm und Rektım, im Norden der des Oesophagus viel häufiger ist, habe ich geschlossen, dass beide örtlich übereinstimmenden Tatsachen die gleiche Ursache haben möchten und da diese bei den Wirten jedenfalls der Missbrauch von Bier im Süden, von Wein und Branntwein im Norden ist, dass' dieser Missbrauch auch in ursächlichem Zusammenhang mit dem betreffenden Krebsvorwiegen bei der ganzen Bevölkerung steht. Wie die Einwirkung von Wein und Branntwein auf die Speiseröhre, so dürfte die Einwirkung des Biers auf den Darm zunächst eine lokale sein. Beim Bier wäre weniger an den Alkohol, als an den Einfluss des Biers im ganzen auf die Darmfunktion zu denken, an die eingeführten Gärungserreger, den Hopfengehalt. Die ungünstige Wirkung des Bieres bei Darmatonie und spastischer Obstipation hat Craemer (58) geschildert und in Verbindung damit kann die Ansicht $\mathrm{H}$ ansemanns angeführt werden, dass ein Zusammenhang zwischen Rektumkrebs und chronischer Obstipation besteht.

Endlich hat sich bei meinen erwähnten Untersuchungen ergeben, dass der Alkohol nicht nur direkt lokal einwirkt, denn es fand sich die überraschende Tatsache, dass der Uteruskrebs bei Wirtinnen viel häufiger ist, als bei anderen Frauen. Er macht bei ihnen $30 \mathrm{pCt}$. aller Krebsfälle aus, gegenüber $17 \mathrm{pCt}$. bei der ganzen weiblichen Berölkerung und dies trotzdem dass bei Wirtinnen schon die allgemeine Krebssterblichkeit eine höhere ist.

Bezüglich derHöhe derUebersterblichkeit im Süden an Darm- und Rektumkrebs verweise ich auf das früher Gesagte. Für beide Lokalisationen zusammengenommen ergibt sich für die Stadt bei den Männern eine Mehrung von 100 auf 147, bei den Frauen auf 133, - für das Land bei den Männern auf 142 , bei den Frauen auf 115 . Man darf dabei allerdings nicht die allgemeine Uebersterblichkeit des Südens vergessen, welche aber nicht ganz soviel beträgt. Bezüglich des Oesophaguskrebses möchte ich nur noch einmal erwähnen, dass während er im ganzen im Norden häufiger ist, nur die männliche Bevölkerung auf dem Lande in Südbayern ausnahmsweise mehr befallen ist (s. S. 216). Sollte der Branntweingenuss hier stärker sein? Es war mir leider nicht möglich, mir eine Statistik des Verbrauchs geistiger Getränke in den einzelnen Teilen Bayerns zu verschaffen.

Eine zweite schädliche Lebensgewohnheit, die des Nichtstillens, habe 
ich bereits 1909 im 8. Bande dieser Zeitschrift zuerst bezüglich des Uteruskrebses nachgewiesen. Ein häufigeres Vorkommen des Mammakrebses infolge von Nichtstillen hatte schon früher Bollinger vermutet, ausgehend von der Beobachtung, dass Nichtstillen zur Atrophie der Drüse führe und atrophische Organe, wie der im Leistenkanal stecken gebliebene Hoden eine Disposition zu Krebs zeigen. Um so mehr war ich überrascht über die gegen mein Erwarten gefundene grössere Häufigkeit des Uteruskrebses bei Nichtstillenden. denn bei ihnen ist das in betracht kommende Organ ja in einem ganz entgegengesetzten, hyperämisch-hyperplastischen Zustande. Und wenn man den Einfluss der Schwangerschait zur Erklärung dieses auffallenden Zusammentreffens anziehen wollte, so steht dem entgegen, dass, wenn jene einen direkten Einfluss hat, er bezüglich Uterus und Mamma gerade entgegengesetzt ist. Gastpar und Weinberg sagen (60): Die relative Unfruchtbarkeit der Frauen mit Neubildung der Brustdrüse muss als gesichertes Ergebnis betrachtet werden, und später: es ist der Einfluss der Geburtenzahl auf den Gebärmutterkrebs bei unserem Material nicht deutlich hervorgegangen. Es stimmen aber die Statistiken fast aller Forscher darin überein, dass verheiratete Frauen häufiger an Uteruskrebs erkranken, als Ledige und Witwen (61). Knack (62) hebt besonders die Seltenheit des Uteruskrebses bei Jungfrauen und die auffallende Seltenheit bei verheirateten Nulliparen hervor. Nach Levin (63) spräche auch gegen den Einfluss der Geburt die praktische Immunität der amerikanischen Indianerinnen gegen Uteruskrebs.

Noch richtiger als die frühere ist die folgende Berechnung der Sterblichkeit nach der bayerischen Durchschnittsbevölkerung und gestützt auf grössere Zahlen.

Tabelle IX.

\begin{tabular}{|c|c|c|c|c|}
\hline & $\begin{array}{c}\text { Sterblichkeit } \\
\text { an Krebs von } \\
\text { Uterus u. Ovarien }\end{array}$ & $\begin{array}{c}\text { Durch- } \\
\text { schnittliche } \\
\text { Stilldauer }\end{array}$ & $\begin{array}{c}\text { Still- } \\
\text { häufigkeit }\end{array}$ & $\begin{array}{l}\text { Sterblichkeit } \\
\text { an } \\
\text { Mammakrebs }\end{array}$ \\
\hline $\begin{array}{l}\text { Oberbayern . . . . } \\
\text { Schwaben . . . . . } \\
\text { Niederbayern. . . . } \\
\text { Mittelfranken. . . } \\
\text { Unterfranken . . . } \\
\text { Oberpfalz . . . . . } \\
\text { Pfalz . . . . . } \\
\text { Oberfranken . . . }\end{array}$ & $\begin{array}{l}794 \\
589 \\
587 \\
559 \\
372 \\
362 \\
286 \\
285\end{array}$ & $\begin{array}{l}0,9 \text { Monate } \\
0,8 \quad " \\
1,0 \quad " \\
3,4 \quad " \\
4,7 \quad " \\
3,1 \quad " \\
6,4 \quad " \\
6,5 \quad "\end{array}$ & $\begin{array}{l}29,3 \text { pCt. } \\
29,2 " \\
26,3 " \\
58,8 " \\
83,4 " \\
55,7 " \\
84,7 " \\
89,6 "\end{array}$ & $\begin{array}{l}298 \\
280 \\
238 \\
300 \\
217 \\
201 \\
118 \\
152\end{array}$ \\
\hline
\end{tabular}

Auch aus Tafel $V$ ersieht man deutlich das entgegengesetzte Verhältnis von Stilldauer und Stillhäufigkeit einerseits und Häufigkeit des Uterus-, in geringerem Masse auch des Mammakrebses andererseits, wobei nur die Oberpfalz eine nach dem früher Gesagten erklärliche Ausnahme macht. 
Man kamn nun einwenden, dass der Krebs im allgemeinen in Südbayern (mit geringerer Stillhäufigkeit) überhaupt öfter vorkommt. Um diesen Einwand zu beseitigen, habe ich Stillhäufigkeit und Vorkommen des Uteruskrebses der einzeInen Regierungsbezirke mit Oberfranken, dieses $=100$ gesetzt, verglichen nach Abzug der Uebersterblichkeit des Krebses im ganzen, d. h. aller Lokalisationen. Das Ergebnis zeigt folgende Tabelle.

\begin{tabular}{|c|c|c|c|c|c|c|c|c|}
\hline & 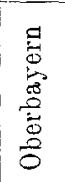 & 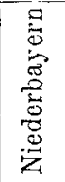 & 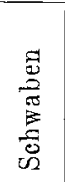 & $\frac{\pi}{\overbrace{\overparen{2}}^{2}}$ & 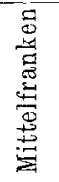 & $\frac{\pi}{2}$ & 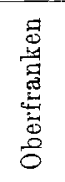 & 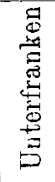 \\
\hline 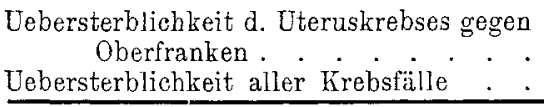 & $\begin{array}{r}279 \\
157 \\
\end{array}$ & $\begin{array}{l}207 \\
118 \\
\end{array}$ & $\begin{array}{l}207 \\
154 \\
\end{array}$ & $\begin{array}{l}127 \\
109 \\
\end{array}$ & $\begin{array}{l}196 \\
129 \\
\end{array}$ & $\begin{array}{l}101 \\
117 \\
\end{array}$ & $\begin{array}{l}100 \\
100 \\
\end{array}$ & $\begin{array}{l}131 \\
116 \\
\end{array}$ \\
\hline Verbleibender Unterschied & 122 & 89 & 53 & 18 & 67 & $|-16|$ & - & 15 \\
\hline
\end{tabular}

Auch diese Rechnung zeigt im ganzen einen entgegengesetzten Parallelismus.

Für die Stadt und das Land ist das Ueberwiegen des Uteruskrebses (hier ohne den Eierstockkrebs berechnet) in Südbayern nahezu gleich gross. Es beträgt in der Stadt 2131/2, auf dem Lande 218, während die Stillhäufigkeit in ganz Bayern in Stadt und Land ziemlich gleich angegeben wird (64). Diese Angaben der Stillhäufigkeit je nach Stadt und Land scheinen aber sehr unvollständig zu sein.

Rosenfeld hält überhaupt die Angaben über Stillhäufigkeit und Stilldauer für so unzuverlässig, dass er statt dessen eine Vergleichung der Uteruskrebshäufigkeit mit der Sänglingssterblichkeit vorschlägt. Damit kommt man rom Regen in die Traufe. Die Säuglingssterblichkeit hängt ausser vom Stillen von so vielen anderen Momenten ab, namentlich von der Art der künstlichen Ernährung und sonstigen Pflege, von Wohnung und Armut (Groth), dann von der Sommerhitze, vielleicht auch vom. Sommerregen (65) ab, dass von vornherein zu erwarten ist, dass diese Vergleichung nur unrichtiger sein kann. Ich habe sie übrigens auch ausgeführt; wie zu erwarten war, gab sie nur ähnliche Beziehungen insofern, als mit Ausnahme von einem alle Regierungsbezirke mit hoher Krebssterblichkeit auch Uteruskrebs ïber dem Mittel, diejenigen mit geringer Sterblichkeit Uteruskrebs unter dem Mittel hatten.

Eine volle Bestätigung des Einflusses des Nichtstillens auf Vermehrung der Fälle von Uteruskrebs sehe ich in den Untersuchungen von Soegard (65). Danach macht der Uteruskrebs im südlichen Bergenhus nur $2,3 \mathrm{pCt}$. aller dortigen Krebsfälle aus. Es kommen $15^{1} / 2$ Fälle auf 1 Million Gesamtbevölkerung (in ganz Bayern 50, also 3 mal soviel) trotz der hohen Geburtsziffer, welche die Entwicklung des Uteruskrebses zu begünstigen scheint. 
Soegard erklärt diese Seltenheit mit der Seltenheit syphilitischer Erkrankungen. Letztere können aber keinesfalls ron durchgreifender Wirkung sein; denn es gibt in Südbayern Bezirke mit sehr häufigem Uteruskrebs ohne häufige Syphiliserkrankungen. Dagegen ist das Stillen in Norwegen wie in Schweden und Dänemark noch allgemein, auch in den höheren Ständen und wird oft bis in das 2. und 3. Jahr fortgesetzt.

Ich führe nur noch an, dass die Sterblichkeit an Uteruskrebs in Sïdbayern auch grösser ist, als in Schottland, England und der Schweiz, verweise aber auf den 8 . Band dieser Zeitschrift, wo ich auch darauf aufmerksam gemacht habe, dass noch andere Einflüsse zum Uteruskrebs disponieren können, welche nicht notwendige, aber häufige Folgen der Kultur sind, wie z. B. verbreiteter künstlicher Abortus, an den man denken mag, wenn man aus einer Angabe von Mc Connell auf Häufigkeit des Krebses der weiblichen Geschlechtsorgane in den Vereinigten Staaten schliessen will.

Es sind ron mir nach dem Vorausgegangenen nunmehr zwei Lebensgewohnheiten nachgewiesen worden, welche einen solchen Einfluss auf die Häufigkeit des Krebses haben, dass sie diese für ganze Landesteile und Länder erhöhen können. Diese Folgerung gilt uneingeschränkt für diejenigen, welche wie Rosenfeld (66) , sich gezwungen sehen, die Krebsstatistik in Unterabteilungen je nach dem Primärsitz der Neubildung zu zerspalten, weil die Bedingungen für die Entstehung eines Uteruskrebses andere sind als für die Entstehung des Mammakrebses und wir uns durch die statistische Zusammenfassung aller Carcinome den Weg zur Erforschung ihrer Natur selbst verrammeln."

So einfach liegt die Sache nicht. Nan muss zur Entstehung des Krebses, weun nicht nach $\mathrm{C} z$ erny 3 , doch mindestens 2 Bedingungen annehmen. Die Krrebsentwicklung ist nach Hansemann (6T) eine Resultante aus dem äusseren Reiz und der Disposition. Die Krebshäufigkeit hängt also von 2 Grössen oder richtiger ron 2 Summen von Grössen ab und wenn wir einen Reiz, einen Koeffizienten ausschalten, wie z. B. den Alkoholmissbrauch, so ist damit nicht gesagt, dass von den Menschen, welche dadurch z. B. von Oesophaguskrebs bewahrt bleiben, nicht so und soriele später an einem anders lokalisierten Krebs sterben. Es ist zu berücksichtigen, dass durch dieses Verschontbleiben nicht nur zahlreiche Menschen ein höheres Alter erreichen, in dem sie an und für sich leichter dem Krebs anheimfallen, sondern dass dies gerade Menschen sind, welche, wie ihr nach unserer Annahme nur durch Vermeidung des Reizes erfolgtes Verschontbleiben beweist, zu Krebs disponiert sind.

Für diesen Schluss spricht auch folgende Ueberlegung. Scheiden wir die Todesfälle durch Uterus- und Mammakrebs ab, so beträgt für alle anderen Krebslokalisationen die weibliche Sterblichkeit gegenüber der männlichen in Südbayern 84, in Nordbayern 88 pCt. Es liegt doch nahe, 
dass dieser geringere Unterschied zu gunsten der Frauen gegenüber den Männern in Nordbayern Folge davon ist, dass in Nordbayern weniger Frauen an Krebs von Uterus und Mamma und deshalb mehr, um 4 pCt., an anderen Krebsen sterben.

Disposition und Reiz sind jedenfalls, wie gesagt, Summen von Grössen. Die Disposition kann eine lokale sein; daneben gibt es aber, wie eingangs erwähnt wurde, eine allgemeine Disposition, wie die des Alters und welche auch unter Anderen Albrech (68) annahm. Dass es Reize rerschiedenster Art gibt, wird allgemein zugegeben.

Solange wir die Grösse der Koeffizienten, der lokalen und allgemeinen Disposition und der Reize nicht kennen, können wir nur sagen: die tatsächliche Ersparung an Krebstoten muss sich bewegen zwischen 0, wenn der Wert des weggefallenen Reizes gegenüber den übrigen Koeffizienten verschwindend klein ist und dem vollen Betrag der verschonten Leben, wenn die anderen Koeffizienten nicht in Betracht kämen.

In unserem Falle würden, wenn in Südbayern nicht 2,3 mal soviel an Krebs der weiblichen Geschlechtsorgane, nicht 1,8 mal soviel an Mammakrebs stürben, als in Nordbayern, sondern nur ebensoviele, von den 3019 Toten der ersteren und den 1218 der zweiten Lokalisation 1313 bzw. 676, zusammen 1989 d. h. 14.6 aller weiblichen Krebstoten weniger sterben.

Ziehen wir davon die oben errechneten $4 \mathrm{pCt}$. der Uebersterblichkeit in Nordbayern ab, so blieben noch 10,6 pCt., welche in Südbayern im besten Falle gerettet werden könnten und dann wäre das Verhältnis der weiblichen Krebssterblichkeit in Südbayern gegenüber Nordbayern nicht mehr 136, sondern noch 126: 100.

Diese Berechmung soll natürlich nur Möglichkeiten andeuten und zugleich zahlenmässiges Denken in der Aetiologie fördern helfen. Sie zeigt aber jedenfalls, dass die gefundenen schädlichen Lebensgewohnheiten wohl einen Teil des rerschiedenen örtlichen Krebsvorkommens, aber keinesfalls die ganze Uebersterblichkeit in Südbayern restlos erklären können.

Wir haben erst begonnen, Einsicht in diese Verhältnisse zu gewinnen; es ist deshälb zuzugeben, dass wir noch mehr solcher Koeffizienten kennen lernen werden und dass diese vielleicht einmal die Verschiedenheit des örtlichen Krebsvorkommens werden erklären kömmen; aber es erscheint mir dies nicht wahrscheinlich.

Ich habe schon vor Jahren den Einfluss von Boden und Haus als die wahrscheinliche Ursache der grossen Krebshäufigkeit in Süddeutschland, der Schweiz und dem angrenzenden Oesterreich zu erweisen gesucht (69), und dass beide hauptsächlich durch ihre Feuchtigkeit, der Boden wahrscheinlich auch durch seine chemische Zusammensetzung (Ton), vielleicht auch grösseren Reichtum an organischen Stoffen; im Alpenvorland besonders durch die weitverbreitete Lehmdecke und unter Mitwirkung der dortigen 
hohen Niederschlagsmenge einwirken. Für die Feuchtigkeit ist allerdings bezüglich Boden und Haus der wirkliche Nachweis jedesmal zu verlangen; ihre blosse Vermutung bei Ansiedelung an Teichen und Flüssen genügt nicht.

Auf der Tafel VI bezeichnet die Linie des Laufes der Donau zugleich die fast genau mit ihr zusammenfallende Nordgrenze der Tertiärformation, einschliesslich Diluvium und Alluvium, welche das nördliche Vorland der Alpen bedeckt. Die hohe Krebssterblichkeit Südbayerns schneidet im Osten fast genau mit dieser Linie ab, während im Westen ein zusammenhängender Streifen (worüber später), nämlich die Bezirksämter Nördlingen und Donauwörth, dariiber hinausreicht, ebenso wie an der fortgesetzten Donau- und Tertiärgrenze in Wiirttemberg das Amt Ehingen, in Baden die Kreise Säckingen und Waldshut. Ich muss in dieser Beziehung auf meine frühere Arbeit vom Jahre 1902 verweisen.

Es war meine Absicht, namentlich diese Verhältnisse näher zu bearbeiten. aber die Gewissheit, dass wir für derartige Untersuchungen im ganzen noch nicht die nötigen Unterlagen besitzen, liess mich daron abstehen. Lntersuchungen im grossen würden in Bayern nur wieder wie früher die grössere Krebshäufigkeit im Tertiärlande gegenüber dem Buntsandstein, Muschelkalk usw. der geologischen Karten nachweisen, aber damit ist nichts Sicheres bezüglich der für den Arzt massgebenden obersten Bodenschichten erwiesen. Es fehlen noch überall durchgehende Feststellungen dieser deckenden Bodenschichten in bezug auf chemische, physikalische, namentlich Feuchtigkeitsrerhältnisse, wie sie Emmerich verlangt, und solange dies der Fall ist, kommt man in dieser frage nicht leicht weiter. Untersuchungen im Detail, welche besonders notwendig wären, sind deshalb bis jetzt kaum möglich, weil, wie Emmerich nachwies, in einem und demselben Dorfe, ja auf kleinste Entfernungen innerhalb desselben die rerschiedenste Beschaffenheit der Bodendecke sich rorfinden kanu. Man müsste in das kleinste Detail eingehen können. So ist es demn begreiflich, dass Werner (70) bei sẹnen eingehenden Lntersuchungen keinen Einfluss der geologischen Beschaffenheit des Bodens auf die Krebshäufigkeit finden konnte. Er bezeichnet es ïbrigens auch als auffällig, dass in einigen Diluvial- und Miozängebieten die Zahl der krebsreichen Gemeinden besonders hoch, in den Gneisgebirgen aber im allgemeinen niedrig ist. Er schliesst aber: "Wohl sind einige geologische Formationen im Durchschnitt krebsreicher als andere, doch finden sich in jeder einzelnen so grosse Differenzen der Carcinommortalität, dass das Vorhandensein einer bestimmten Gesteinsart nicht zur Erklärung der häufigeren Entstehung des Ǩrebses herangezogen werden kann“ und er fügt mit Recht bei: „Lohnender wäre es vielleicht, Untersuchungen ïber die chemische Zusammensetzung der oberfächlichen Bodenschichten systematisch durchzuführen für jene Orte, in denen abnorm viele oder abnorm wenige Krebsfälle nachgewiesen wurden. 
(Uebrigens kommt man dabei leicht in die statistischen Gefahren der zu kleinen Zahlen.)

Werner, der die Differenzen der Krebsdichte in den verschiedenen Gegenden Badens als erwiesen hält, aber keinen Finfluss als sichere Ursache dieser Erscheinung feststellen konnte, kommt daher zu dem Ergebnis: „Die räumliche und zeitliche Verteilung der Krebsirequenz deutet vielmehr direkt auf einen ursächlichen Zusammenhang mit einem äusseren an diesem Orte haftenden Agens." Wo aber könnte dieses Agens sonst sitzen, ausser in Boden und Haus?

Es seien hier nur noch einige Unstimmigkeiten und gegen die Bodentheorie namentlich von Rosenfeld gemachte Einwände besprochen. Ich habe schon früher darauf hingewiesen, dass das, was die geologische Karte mit derselben Farbe bezeichnet, physikalisch und chemisch sehr verschieden sein kann, und dass umgekehrt verschiedene geologische Formationen dieselbe physikalisch-chemische Beschaffenheit haben können. Ich habe dort auch bemerkt, dass die Krebshäufigkeit im Süden des Schwarzwalds durch die Verbreitung von Ton, Tonmergel, tonigem Sandstein, namentlich bei Waldshut und Säckingen, diejenige in den Alpen Salzburgs und Nordtirols durch den grossen Gehalt von Rhät und verschiedenen Triasbildungen an Tonen und Mergeln erklärt werden könnten und habe damit zum roraus auf einen Einwand Rosenfelds geantwortet.

Nach der frïheren Arbeit fand sich einerseits keine hohe Krebssterblichkeit auf dem Tertiärlande Südbayerns an der Nordgrenze im Osten. andererseits wird das Tertiärland auch nach der heutigen Karte an der Nordgrenze im Westen von der hohen Sterblichkeit überschritten. Der erste Widerspruch zwischen Theorie und Wirklichkeit ist aber tatsächlich nicht vorhanden, wie die getrennte Berechnung für die südlich der Donan und die nördlich von ihr liegenden Gemeinden der betreffenden Bezirksämter bewies. Die 4 Bezirksämter haben nämlich folgende Krebssterblichkeit:

Straubing . . südlich der Donau 2385, nördlich 797

$\begin{array}{lllllll}\text { Deggendorf . } & " & " & " & 2721, & & 2032 \\ \text { Vilshofen . . } & " & , & " & 2095, & & 1024 \\ \text { Passau . . . . } & . & , & " & 2224, & , & 1407\end{array}$

Wie die südlichen, so schliessen sich also auch die nördlichen Teile der Krebshäufigkeit der benachbarten Bezirksämter an. Die nördlichen haben dieselbe niedrige Sterblichkeit wie das sonstige nördliche Niederbayern und die anstossende Oberpfalz. Ich möchte diese Mindersterblichkeit allerdings zum Teil durch die wahrscheinlich unvollständigere Erhebung, zum Teil aber auch dem Gneis- und Granitboden zuschreiben.

Der zweite Widerspruch wird dadurch erklärt, dass nach genaueren. Karten das Tertiär im Westen Bayerns nicht an der Donau Halt macht. Das Bezirksamt Donauwörth hat vielmehr im östlichen Teile nahezu ganz 
tertiären Boden und ausserdem decken hier, wie im Bezirksamt Nördlingen, besonders im Ries, jüngere von der südbayerischen Hochfläche herkommende Ablagertungen, vorzugsweise die lehmige Albüberdeckung, den Jura. Gümbel sagt von dieser Gegend: „Im Gebiete des Frankenjura herrschen durchweg Mergelböden als Umbildungsprozesse der Ueberdeckungsablagerungen oder der mergeligen Juragesteine vor." - Die beiden vermeintlichen Widersprüche geben also vielmelır nach ihrer Erklärung eine Bestätigung der Regel.

Eine Einwendung Rosenfelds lautet: „dass die Ortschaften gleicher geologischer Formation und wahrscheinlich gleicher Bodenfeuchtigkeit von \pm um München herum gelegenen Bezirksämtern eine sehr verschiedene Krebssterblichkeit je nach dem Bezirksamt haben, was zumindest auf noch andere Einflüsse als die Bodenfeuchtigkeit hindeutet." Diese Vermutung trifft nicht zu; nur das stark befallene Bezirksamt Dachau hat reinen Tertiärboden und weiter ist zu erwidern, dass die einzelnen Teile anch der anderen drei Bezirksämter keineswegs gleiche Bodenbeschaffenheit und Bodenfeuchtigkeit haben (31). Das beweist schon das durch Pettenk ofer bekannte Dorf Aubing. Emmerich fand, dass der Untergrund dieses. Dorfes im Osten Kies, in Westen Lehm ist, und dass sich eine Verschiedenheit des Grundwasserspiegels zwischen 1 und $5 \mathrm{~m}$ rorfindet.

Rosenfeld wirft weiter ein: „Es deutet auf ganz andere Ursachen (nämlich als den Boden), dass die politische Grenze zwischen Baden und Württemberg auch die scharfe Grenze zweier Gebiete verschieden hoher Krebssterblichkeit ist." In diesem Falle macht aber die politische zugleich auch die ge ologische Grenze, von Neuenbürg nach Süden, zwischen dem badischen Urgebirge (Granit und Gneis) und dem württembergischen Buntsandstein und Muschelkalk, so dass vielmehr diese Beobachtung gerade zugunsten der Bodentheorie rerwendet werden kam.

Es ist andererseits eine bedeutsame Bestätigung derselben, dass, wie ich schon vor Jahren hervorgehoben habe, so häufig hohe oder auch niedrige Krebssterblichkeit sich ïber die Grenzen benachbarter Länder hinwegsetzt. Ich verweise nur nochmals auf die hohe Krebssterblichkeit des nördlichen Alpenvorlandes von Wien bis Genf, also in zusammenhängenden Teilen Oesterreichs, Bayerns, Württembergs, Badens und der Schweiz, auf die Maxima derselben in Salzburg, itbergreifend auf Berchtesgaden, und in Bayerisch Schwaben, uibergreifend auf den württembergischen Donaukreis, andererseits auf die geringe Sterblichkeit in der Oberpfalz und Niederbayern, zusammenlängend mit der im anstossenden Böhmen.

Rosenfeld führt selbst an, dass die Franken in Bayern, da wo sie an ein von den andern Volksstämmen bewohntes Gebiet hoher Krebssterblichkeit anstossen, auch eine höhere Krrebssterblichkeit, wo sie an Gebiete niederer Krebssterblichkeit grenzen, eine niedrige Krebssterblichkeit haben. 
"Es macht dies tatsächlich den Eindruck, dass der Satz, die Krebshäufigkeit sei an die Lokalität gebunden, zu Recht besteht" (S. 28).

Anschlüsse ron Ländern bezüglich ähnlicher Krebshäufigkeit sind selbstverständlich bedeutsamer als Differenzen, denn diese können leicht durch verschiedenartige Erhebung, Verschiedenheit in Inanspruchnahme der Aerzte, Volksgewohnheiten usw. hervorgerufen werden. Wenn man allerdings zu jedem Vergleiche gleiche soziale Verhältnisse in den benachbarten Ländern verlangte, so würde dies Vergleichungen meist unmöglich machen - oft selbst im eigenen Land, wie zwischen Ost- und Rheinpreussen, dem Erzherzogtum Oesterreich und Istrien oder Galizien.

Rosenfeld hat weitere Zweifel: „ein Abflachen der Krebshäufigkeit von einem Zentrum aus, wie in Südbayern spricht mir weniger zugunsten der Bodenhypothese, als dass es auf mich den Eindruck allmählich abklingender Volksgewohnheiten als ätiologischer Faktor hat." - In Südbayern ist aber nicht ein einziges Zentrum, sondern 2 Zentren, eines bei Augsburg im nördlichen Schwaben and angrenzenden Oberbayern und ein zweites in Berchtesgaden nebst Salzburg (siehe die neue Karte). Beide sind allerdiugs verbunden durch einen breiten Landstrich mit hoher Krebssterblichleit, der fast ganz Oberbayern in sich begreift. Nur im Süden sind Bezirksämter mit geringerer Sterblichkeit; München, Wolfratshausen, Weilheim, Tölz, Füssen und Schongau. Eine sichere Erklärung für diese Abnahme ist auf Grund der vorliegenden geologischen Tatsachen nicht möglich. Ich möchte aber doch erwähnen, dass die Hauptmasse des die südlichen Bezirksämter deckenden Bodens Gerölle und Schutt bilden, welche in den Flussgebieten von Isar und Inn nach ihrem Ursprungsgebirge ganz verschieden sind. Aber kein Kenner der Bevölkerung dieses Landstriches, der übrigens im Süden wieder an stark von Krebs befallene Bezirke: Kufstein, Innsbruck, Kitzbichl anstösst, wird Volksgewohnheiten auffinden, welche ihm im Gegensatz zu den krebsreichen Bezirken Oberbayerns eigentümlich sind.

Eine teilweise Erklärung für das allmähliche Lebergehen der Krebshäufigkeit auf benachbarte Bezirke liegt in den immer zahlreicher werdenden Wanderungen ihrer Bewohner, namentlich in Industriegegenden, selbst wenn man davon absieht, dass bei Annahme parasitärer Entstehung des Krebses auch bei kurzdauernden Aufenthaltsveränderungen die Keime der Krankheit erworben werden könnten (Rosenfeld S. 13). Dass in den Städten, abgesehen von ausnahmsweisen Verhältnissen, wie z. B. in Passau, in der Regel eine Topographie des Krebses herzustellen nutzlos ist, zeigt eine Angabe (73), wonach von der Einwohnerschaft Diisseldorfs im Jahre 1905 (1/4 Million) der fünfte Teil erst im Laufe dieses Jahres zugezogen war.

Schliesslich möchte ich noch auf die Angaben Werners über das Wohnhaus einiges bemerken. Werner sagt: Die Differenzen des Schwarzwälder Bauernhauses und der fränkischen Hofanlage bei gleichartigen Einteilung der Stallungen und Vorratsräume, meist auch des Baumaterials . . ., 
wobei allerdings das Schwarzwaldhaus und der fränkische Hofbau in vieler Beziehung volle Gegensätze darstellen, erklären die Unterschiede der Krebsdichte nicht (S. 278). Dabei scheint mir doch die Frage nicht scharf gestellt. Ich sehe als wichtigste Eigenschaft des Hauses für die Krebsätiologie die Feuchtigkeit an und diese wird sich vor allem richten nach dem Untergrund und dem Baumaterial, dann nach dem Klima, besonders der Menge der Niederschläge, der Luftfeuchtigkeit, ferner nach der Bauart: Unterkellerung, An- und Einbau von Ställen, Aborten und Düngerstätten, welche ihren flüssigen Inhalt direkt den Hausmauern mitteilen oder durch Versickerung den Untergrund gründlich durchfeuchten, endlich nach der Benutzung: Ueberfüllung. Waschen usw. (73). Es geht aus obigen Worten Werners nicht hervor, ob in erster Linie der Feuchtigkeitsgrad der Häuser untersucht wurde, oder dieser nicht hinter nebensächlichen Umständen in den Hintergrund getreten ist. Sind unter den „in vielen Beziehungen in vollen Gegensätzen" stehenden Haustypen vielleicht gerade solche Gegensätze, welche den Feuchtigkeitsgrad beeinflussen?

Es dürfte bei den so nötigen Detailuntersuchungen sich vor allem lohnen, die Feuchtigkeit der Häuser und des unter und neben ihnen liegenden Bodens für ganze Ortschaften zu untersuchen.

Von neueren Untersuchungen über die Krebshäufigkeit, welche allerdings fast nur nach einer mangelhaften Methode, $d . h$. mit Nichtberïcksichtigung des Alters und der zugezogenen Kranken angestellt wurden, seien kurz einige angefülhrt.

Bertillon hat 1911 auf Grund der damals seit 3 Jahren veröffentlichten Berichte des Ministerium des Innern übereinstimmend für die Land-, wie für die Stadtbevölkerung sich dahin aussprechen können (74): Lnsere Karte erinnert an die geologische Karte ron Frankreich. Das Viereck zwischen dem Meer, Caen, Anger, Dijon und Mézières, das 2 bis 3 bis 4 mal soviel befallen ist als der Osten, ähnelt sehr dem Kreidebecken, dessen Zentrum Paris ist, aber weicht doch von ihm ab, denn die (ebenfalls stark befallenen) Departements Pas-de-Calais, le Nord usw. gehören nicht dazu. Bertillon irrt sich aber merkwürdigerweise. Jene Departements gehören auch noch zu dem grossen Landstrich im Norden Frankreichs, welcher der Kreide und in dem von ihr umschlossenen Zentrum dem Tertiär angehört, so dass wir hier sogar ein recht weitgehendes Zusammentreffen von Krebsdichte und 2 geologischen Formationen vor uns haben. Die grosse Krebshäufigkeit in Frankreich überschreitet allerdings diese beiden Formationen nach Südosten, wo sie sich über den Jura (Dep. Yonne und Cotes d' Or), dann nach Süden über die Departements auf dem linken Rhoneufer, besonders Rhône und Isère erstreckt. Die letztgenannten Departements gehören übrigens auch ganz oder zu grossem Teil Tertiär und Kreide an.

Es beruht vielleicht auf keinem Zufall, dass die grösste Krebssterblichkeit 
in Italien auf der Karte der Internationalen Hygiene-Ausstellung (nach Prinzing) die Provinzen Parma, Florenz und Siena betrifft, von denen die erste zum grossen Teil, die beiden letzten ganz tertiär sind. Ich habe das gleiche Verhältnis übrigens schon vor Jahren ebenfalls gefunden.

Ich möchte als Ergebnis der vorstéhenden Untersuchungen zusammenfassen:

1. Die Krebshäufigkeit in Bayern ist - was seit mehr als 2 Jahrzehnten nachgewiesen ist - höher im Süden, als im Norden, wie gleicherweise seit 2 bis 3 Jahrzehnten immer wieder dieselben Unterschiede des örtlichen Vorkommens des Krebses in der Schweiz, in Baden, Württemberg und Oesterreich bestehen.

2. Diese Unterschiede sind für die einzelnen Organlokalisationen, aber auch für die Gesamtsterblichkeit an Krebs durch Volksgewohnheiten zum Teil zu erklären; wahrscheinlich beruhen sie aber in der Hauptsache auf örtlichen Einflüssen des Bodens und Hauses.

3. Es ist nunmehr möglich, die Krebshäufigkeit zu vermindern durch den Kampf gegen Alkoholmissbrauch und Nichtstillen. Der Kampf gegen diese zwei sittlichen Sünden kann von jetzt an um so nachdrücklicher geführt werden, als er sich zugleich gegen sie als hygienische Sünden, welche das eigene Wohl schädigen, richten kann.

Es gehört deshalb künftig in alle Merkblätter zur Bekämpfung des Krebses vor allem die eindringlichste Lehre, dass zur Vermeidung der schrecklichen Krankheit auch Vermeidung des Alkoholmissbrauchs und des Nichtstillens notwendig ist.

\section{Literaturverzeichnis.}

1. Weinberg und Gastpar, Zeitschr. f. Krebsforschung. 1904. Bd. 2. Heft 3.

2. Ref. Münchener med. Wochenschr. 1912. S. 2426.

3. Kolb, Zeitschr. f. Hygiene. 1902. Bd. 40. S. $373 \mathrm{ff}$.

4. Laspeyres im Zentralbl. f. allgem. Gesundheitspflege. 1901. S. 370.

5. Zeitschr. f. Krebsforschung. Bd. 8 und 9.

6. Zeitschr. f. Krebsforschung. Bd. 13. S. 558.

7. Zeitschr. f. Krebsforschung. 1912. Bd. 12. Heft 1.

8. Die Naturwiśsenschaften. 1913. 1. Jahrg. Heft 7.

9. Han semann in Zeitschr. f. Geburtshilfe. Bd. 74. S. 154.

10. Zeitschr. f. Krebsforschung. Bd. 13. S. 591.

11. Zentralbl. f. Krebskrankheiten. Bd. 6. S. 153.

12. Zeitschr. f. Hygiene. 1902. Bd. 40. - Zeitschr. f. Krebsforschung. Bd. 8.

13. Zeitschr. f. Hygiene. Bd. 40. S. 386.

14. Edinburgh medical Journal vom 30. Januar 1903.

15. Semain $\theta$ médicale. 1902. No. 37.

16. Nordisches Medizinisches Archiv. 1901. 2. Abt. 
17. Siehe namentlich die Bayerischen Sanitätsberichte seit den $90 \mathrm{er}$ Jahren.

18. Schweizerische Statistik. 1903. Liefg. 137 (betrifft die Jahre 1881-1890). Zeitschr. f. schweizerische Statistik. 1908. 44. Jahrg. Bd. 2 (für 1901-1906).

19. Oesterreichische Statistik. Bd. 50 (für 1887 und $1888 \mathrm{ff}$.).

20. Zeitschr. f. schweizerische Statistik. 1900. S. 332 (für die Jahre 1889-1898).

21. Zeitschr. f. Hygiene. 1902. Bd. 40.

22. Oesterreichisches Sanitätswesen. 1902. Nr. 45. Beilage.

23. Zentralbl. f. offentl. Gesundheitspflege. 1904. 23. Jahrg. - Zeitschr. f. Krebsforschung. 1907. Bd. 5.

24. Zeitschr. f. schwoizerische Statistik. 1906. 42. Jahrg.

25. Werner, Statistische Untersuchungen über das Vorkommen des Krebses in Baden. Tübingen 1910. (Betrifft die Jahre 1883-1907). - Resultate der Badischen Krebsstatistik 1910.

26. Rosenfeld, Wiener klin. Wochenschr. 1913. Nr. 38.

27. Wolff, Die Lehre von der Krebskrankheit. 3. Teil. S. 44. Jena 1913.

28. Klinisches Jahrbuch. 1902. Bd. 8. S. 352.

29. Ref. im Sonderkatalog der Statistik der Dresdener Ausstellung. 1911. S. 102.

30. Zeitschr. f. Krebsforschong. 1904. Bd. 2. S. 248 und 1912. Bd. 11. S. 306/7.

31. Kolb, Der Einfluss von Boden und Haus auf die Häufigkeit des Krebses. München 1904. S. 4-6.

32. Zeitschr. f. Krebsforschung. Bd. 9. Heft 3.

33. WoIff (s. Nr. 27), 2. Teil. S. 835 .

34. Cohnheim, Vorlesungen über allgemeine Pathologie. 1882. Bd. 2.

35. Sächsischer Sanitätsbericht für 1911. S. 201 und 202.

36. Münchener med. Wochenschr. 1914. S. 147.

37. Versammlung der deutschen anthropolog. Gesellschaft. 1899.

38. La Semaine médicale. 1902. No. 39.

39. Wolff (s. Nr. 27), 3. Teil. S. 39,42 u. 43.

40. Communications statistiques par le bureau municipal d'Amsterdam. 1911.

41. Renner im Zentralbl. f. Krebskrankheiten. 1910. 6. Jahrg. S. 154.

42. Vierteljahrsschr. f. öffentl. Gesundheitspflege, 1906. Bd. 37. S. 544.

43. Ref. Münchener med. Wochenschr. 1910. S. 1898.

44. Wolff, 2. Teil. S. 100.

45. Nordisches Medizinisches Archiv. 1901. 2. Abt. S. 13.

46. Zeitschr. f. Krebsforschung. 1902. Bd. 2. S. 255.

47. Zeitschr. f. Krebsforschung. Bd. 11. S. 547. - Studies in cancer. Vol. II. New York 1912.

45. Zeitschr. f. Krebsforschung. Bd. 12. S. 128.

49. Münchener med. Wochenschr. 1912. S. 2426. - Imperial cancer research fund. 11. Bericht über die Sitzung vom 24. Juli 1913.

50. Zeitschr. f. Krebsforschung. Bd. 13. S. 501.

51. Biochemical Journal. 1909. Vol. IV. No. 5 and 6.

52. Ref. von Schuchardt im Thüringer Korrespondenzblatt. 1893. S. 447, and 1894. S. $64 \mathrm{ff}$.

53. Preussische Statistik für die Jahre 1895 u. 1896. S. 56.

54. Zeitschr. f. Krebsforsehung. Bd. 5. S. 275.

55. Lancet. 1898. Vol. II. p. 770. 
56. Supplement to the 65 th Annual Report of the Registrar General. 1908. p. CLXXXVI and XLI.

57. Vierteljahrsschr. f. öffentl. Gesundheitspflege. Bd. 38 . S. 557 u. 558.

58. Friedr. Craemer, Vorlesungen über Magen- und Darmkrankheiten. 3. Heft. München 1907.

59. Zeitschr. f. Geburtshilfe. Bd. 74. S. 157 .

60. Zeitschr. f. Krebsforschung. Bd. 2. S. 237.

61. Wolff, Die Lehre von der Krebskrankheit. Bd. 2. S. 963.

62. Zeitschr. f. Krebsforschung. 1912. Bd. 11. S. 470 u. 473.

63. Studies in Cancer. 1912. Vol. 1I. p. 24.

64. Groth in Münchener med. Wochenschr. 1909. S. 164i, und 1908. S. 76.

65. Zeitschr. f. Krebsforschung. 1913. Bd. 13. S. 88.

66. S. oben $\mathrm{Nr}, 26$.

67. 1. c. Nr. 9. S. 152.

68. Zeitschr. f. Krebsforschung. Bd. 4. S. 138.

69. 1. c. Nr. 13 und Nr. 31.

70. Werner, l. c. Nr. 25. a) S. 276 und b) S. 24.

71. Emmerich, Pettenkofers Bodentheorie der Cholera. München 1910. S. $439 \mathrm{ff}$.

72. Wygodzin ski, Wandlungen der deutschen Volkswirtschaft im 19. Jahrhundert.

73. Unglert im Bayerischen Sanitätsbericht für 1907.

74. La Presse médicale. 1911. No. 39. 